PREPARED FOR THE U.S. DEPARTMENT OF ENERGY, UNDER CONTRACT DE-AC02-76CH03073

PPPL-3819

PPPL-3819

UC-70

\title{
Scaling of Cross Sections \\ for Ion-atom Impact Ionization
}

by

I. Kaganovich, B. Startsev, and R. Davidson

June 2003
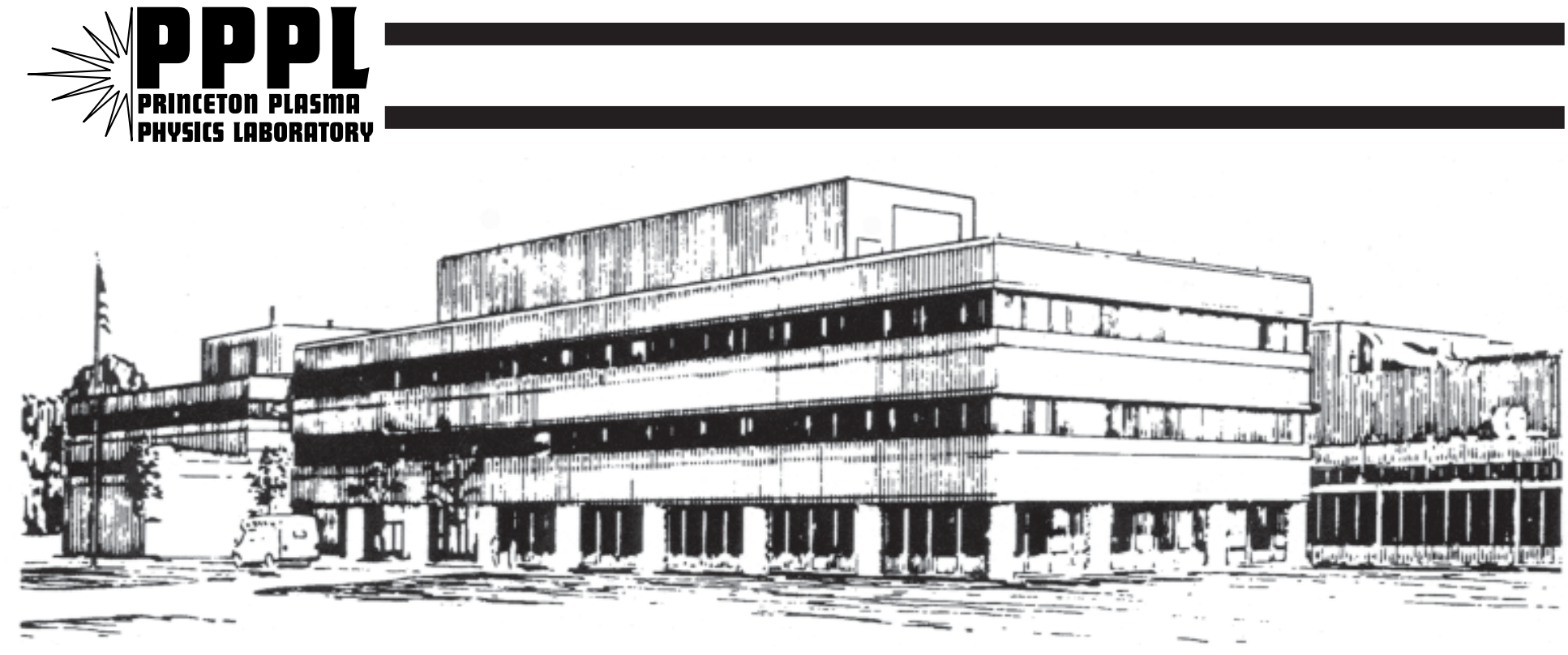

PRINCETON PLASMA PHYSICS LABORATORY PRINCETON UNIVERSITY, PRINCETON, NEW JERSEY 


\section{PPPL Reports Disclaimer}

This report was prepared as an account of work sponsored by an agency of the United States Government. Neither the United States Government nor any agency thereof, nor any of their employees, makes any warranty, express or implied, or assumes any legal liability or responsibility for the accuracy, completeness, or usefulness of any information, apparatus, product, or process disclosed, or represents that its use would not infringe privately owned rights. Reference herein to any specific commercial product, process, or service by trade name, trademark, manufacturer, or otherwise, does not necessarily constitute or imply its endorsement, recommendation, or favoring by the United States Government or any agency thereof. The views and opinions of authors expressed herein do not necessarily state or reflect those of the United States Government or any agency thereof.

\section{Availability}

This report is posted on the U.S. Department of Energy's Princeton Plasma Physics Laboratory Publications and Reports web site in Fiscal Year 2003. The home page for PPPL Reports and Publications is: http://www.pppl.gov/pub_report/

DOE and DOE Contractors can obtain copies of this report from:

U.S. Department of Energy

Office of Scientific and Technical Information

DOE Technical Information Services (DTIS)

P.O. Box 62

Oak Ridge, TN 37831

Telephone: (865) 576-8401

Fax: (865) 576-5728

Email: reports@adonis.osti.gov

This report is available to the general public from:

National Technical Information Service

U.S. Department of Commerce

5285 Port Royal Road

Springfield, VA 22161

Telephone: $1-800-553-6847$ or

(703) $605-6000$

Fax: (703) 321-8547

Internet: http://www.ntis.gov/ordering.htm 


\title{
Scaling cross sections for ion-atom impact ionization
}

\author{
Igor D. Kaganovich, Edward Startsev and Ronald C. Davidson \\ Plasma Physics Laboratory, Princeton University, Princeton, NJ 08543
}

(Dated: June 2, 2003)

\begin{abstract}
The values of ion-atom ionization cross sections are frequently needed for many applications that utilize the propagation of fast ions through matter. When experimental data and theoretical calculations are not available, approximate formulas are frequently used. This paper briefly summarizes the most important theoretical results and approaches to cross section calculations in order to place the discussion in historical perspective and offer a concise introduction to the topic. Based on experimental data and theoretical predictions, a new fit for ionization cross sections is proposed. The range of validity and accuracy of several frequently used approximations (classical trajectory, the Born approximation, and so forth) are discussed using, as examples, the ionization cross sections of hydrogen and helium atoms by various fully stripped ions.
\end{abstract}




\section{INTRODUCTION}

Ion-atom ionizing collisions play an important role in many applications such as heavy ion inertial fusion [1], collisional and radiative processes in the Earth's upper atmosphere [2], ion-beam lifetimes in accelerators [3], atomic spectroscopy [4], and ion stopping in matter [5], and are of considerable interest in atomic physics [6]. The recent resurgence of interest in charged particle beam transport in background plasma is brought about by the recognition that plasma can be used as a magnetic lens. Applications of the plasma lens ranging from heavy ion fusion to high energy lepton colliders are discussed in Refs. [6-9]. In particular, both heavy ion fusion and high energy physics applications involve the transport of positive charges in plasma: partially stripped heavy elements for heavy ion fusion; positrons for electron-positrons colliders [9]; and high-density laser-produced proton beams for the fast ignition of inertial confinement fusion targets [11].

To estimate the ionization and stripping rates of fast ions propagating through gas or plasma, the values of ion-atom ionization cross sections are necessary. In contrast to the electron [12] and proton $[13,14]$ ionization cross sections, where experimental data or theoretical calculations exist for practically any ion and atom, the knowledge of ionization cross sections by fast complex ions and atoms is far from complete [16]. While specific values of the cross section for various pairs of projectile ions and target atoms have been measured at a number of energies [17-19], the scaling of cross sections with energy and target or projectile nucleus charges has not been experimentally mapped. When experimental data and theoretical calculations are not available, approximate formulas are frequently used.

The most popular formula for ionization cross sections was proposed by Gryzinski [20]. The web of science search engine [21] shows 457 citations of the paper, and most of the citing papers use Gryzinski's formula to evaluate the cross sections. In this approach, the cross section is specified by multiplication of a scaling factor and the unique function of the projectile velocity normalized to the orbital electron velocity. The popularity of Gryzinski's formula is based on the simplicity of the calculation, notwithstanding the fact that his formula is not accurate at small energies.

Another fit, proposed by Gillespie, gives results close to Gryzinski's formula at large energies, and makes corrections to Gryzinski's formula at small energies [22]. Although more accurate, Gillespie's fit is not frequently used in applications, because it requires a 
knowledge of fitting parameters not always known a priori.

In this paper, we propose a new fit formula for ionization cross section which has no fitting parameters. The formula is checked against available experimental data and theoretical predictions. We also briefly review the most important theoretical results and approaches to cross section calculations in order to place the discussion in historical perspective and offer nonspecialists a concise introduction to the topic.

The organization of this paper is as follows. In Sec.II we give a brief overview of key theoretical results and experimental data. Further details of the theoretical models are presented in Appendices A-C. The new proposed fit formula for ionization cross section is

presented in Sec.III, including a detailed comparison with experimental data, and in Sec.IV the theoretical justification for the new fit formula is discussed.

\section{OVERVIEW OF THE THEORETICAL MODELS AND EXPERIMENTAL DATA FOR IONIZATION CROSS SECTIONS}

There are several theoretical approaches to cross section calculations. These include: classical calculations that make use of a classical trajectory and the atomic electron velocity distribution functions given by quantum mechanics [this approach is frequently referred to as classical trajectory Monte Carlo (CTMC)]; quantum mechanical calculations based on Born, eikonal or quasiclassical approximations, and so forth [16]. All approaches are computationally intensive and the error and range of validity are difficult to estimate in most cases. Therefore, different fittings and scalings for cross sections are frequently used in practical applications.

Most scalings were developed using theories and simulations based on classical mechanics. Classical trajectory calculations are easier to perform compared with quantum mechanical calculations. Moreover, in some cases the CTMC calculations yield results very close to the quantum-mechanical calculations [17]. The reason for similar results lies in the fact that the Rutherford scattering cross section is identical in both classical and quantum-mechanical derivations [23]. Therefore, when an ionizing collision is predominantly a consequence of the electron scattering at small impact parameters close to the nucleus, the quantum mechanical uncertainty in the scattering angle is small compared with the angle itself, and the classical calculation can yield an accurate description [24, 25]. 
In the present analysis, we consider first the stripping or ionization cross section of the hydrogen-like electron orbits (for example one-electron ions), with nucleus of charge $Z_{T}$, colliding with a fully stripped ion of charge $Z_{p}$. Subsequently, we show that the approach can be generalized with reasonable accuracy for any electron orbital, making use of the ionization potential of the electron orbitals. Because different terminology is used in the literature, we call a stripping collision a collision in which the fast ion loses an electron in a collision with a stationary target ion or atom (in the laboratory frame); and we call an ionizing collision a collision in which a fast ion ionizes a stationary target ion or atom [16]. Both cases are physically equivalent to each other by changing the frame of reference, and further consideration can be given in the frame of the atom or ion being ionized.

Atomic units are used throughout this paper with $e=\hbar=m_{e}=1$, which corresponds to length normalized to $a_{0}=\hbar^{2} /\left(m_{e} e^{2}\right)=0.529 \cdot 10^{-8} \mathrm{~cm}$, velocity normalized to $v_{0}=e^{2} / \hbar=$ $2.19 \cdot 10^{8} \mathrm{~cm} / \mathrm{s}$, energy normalized to $E_{0}=m_{e} v_{0}^{2}=2 R y=27.2 \mathrm{eV}$, where $R y$ is the Rydberg energy. The normalizing coefficients are kept in all equations for robust application of the formulas. For efficient manipulation of the formulas it is worth noting that the normalized

velocity is $\left.v / v_{0}=0.2 \sqrt{E[k e V / a m u}\right]$, where $E$ is energy per nucleon in $k e V / a m u$. Therefore, $25 \mathrm{keV} / \mathrm{amu}$ corresponds to the atomic velocity scale. Some papers express the normalized velocity $v / v_{0}$ as $\beta \alpha$, where $\beta=v / c$, and $v_{0} / c=\alpha=1 / 137$. Here, $c$ is the speed of light, and $\alpha$ is the fine structure constant.

For a one-electron ion, the typical scale for the electron orbital velocity is $v_{n l}=v_{0} Z_{T}$. Here, $n, l$ is the standard notation for the main quantum number and the orbital angular momentum [23]. The collision dynamics is very different depending on whether $v$ is smaller or larger than $v_{n l}$.

\section{A. Behavior of cross sections at large projectile velocities $v>v_{n l}$}

If $v>>v_{n l}$, the electron interaction with the projectile ion occurs for a very short time and the interaction time decreases as the velocity increases. Therefore, the ionization cross section also decreases as the velocity increases. In the opposite case $v<<v_{n l}$, the electron circulation around the target nucleus is much faster than the interaction time, and the momentum transfer from the projectile ion to the electron averages out due to the fast circulation. Thus, the cross section decreases as the projectile velocity decreases. This is 
why the cross section typically has a maximum at $v=v_{\max } \sim v_{n l}$, but as we shall see below, $v_{\max }$ also depends on the charge of the projectile.

\section{Thompson's treatment}

In the first treatment, Thompson calculated the ionization cross section in the limit $v>>v_{n l}[26]$. This treatment neglected completely the orbital motion of the target electrons and assumed a straight-line trajectory of the projectile. In this approximation, the velocity kick acquired by the electron during the collision is entirely in the direction perpendicular to the ion trajectory, because the final action of the force along the trajectory cancels out due to symmetry, i.e., the electron velocity change during the approaching phase is equal to minus the electron velocity change during the departing phase. The momentum acquired by the electron $\left(m_{e} \Delta v\right)$ from passing by the projectile moving with the speed $v$ and impact parameter $\rho$ is given by the integral over time of the force perpendicular to ion trajectory $F_{\perp}=e^{2} Z_{p} \rho /\left(\rho^{2}+v^{2} t^{2}\right)^{3 / 2}$, where $t=0$ corresponds to the distance of the closest approach. Time integration of the force yields

$$
\Delta v(\rho)=\frac{2 e^{2} Z_{p}}{m_{e} v \rho}
$$

From Eq.(1) it follows that only collisions with sufficiently small impact parameters result in ionization. The minimum impact parameter for ionization of an initially stationary electron $\left(\rho_{\min }\right)$ is $m_{e} \Delta v\left(\rho_{\min }\right)^{2} / 2=I_{n l}$. During a collision with impact parameter $\rho_{\min }$ the energy transfer from the projectile to the electron is equal to the ionization potential $I_{n l}=Z_{T}^{2} E_{0} / 2$, or $\Delta v\left(\rho_{\min }\right)=v_{n l}$. Substitution of Eq.(1) gives the total ionization cross section $\pi \rho_{\text {min }}^{2}$ $[24,26]$

$$
\sigma^{B o h r}\left(v, I_{n l}, Z_{p}\right)=2 \pi Z_{p}^{2} a_{0}^{2} \frac{v_{0}^{2} E_{0}}{v^{2} I_{n l}} .
$$

Similarly, Eq.(2) can be derived by averaging the Rutherford cross section over all scattering angles leading to ionization. Although the first derivation of Eq.(2) was done by Thompson [26] the formula is frequently referred to as the Bohr formula [16].

\section{Gerjuoy's treatment}

The following treatments account for the effect of finite electron orbital velocity. The most complete and accurate calculations were done by Gerjuoy [27]. He calculated the 
differential cross section $d \sigma / d \Delta E\left(v_{e}, v, \Delta E\right)$ of energy transfer $\Delta E$ in the collision between the projectile ion and a free electron (the target atomic potential was neglected) with given initial speed $v_{e}$ (and arbitrary direction), by averaging the Rutherford cross section over all orientations of electron orbital velocity $\mathbf{v}_{e}$. The total cross section is then calculated by integration over the energy transfer for energies larger than the ionization potential, and weighted by the electron velocity distribution function $f\left(v_{e}\right)$. This gives

$$
\sigma\left(v, I_{n l}, Z_{p}\right)=Z_{p}^{2} \int_{0}^{\infty} \sigma_{I_{n l}}\left(v, v_{e}\right) f\left(v_{e}\right) d v_{e}
$$

where

$$
\sigma_{I_{n l}}\left(v, v_{e}\right)=\int_{I_{n l}}^{\infty} \frac{d \sigma}{d \Delta E}\left(v, v_{e}, \Delta E\right) d \Delta E
$$

A rather complicated analytical expression for $d \sigma / d \Delta E\left(v_{e}, v, \Delta E\right)$ is given in Appendix A. For large projectile ion velocities $\left(v>>v_{n l}\right)$, the differential cross section can be expressed as $[27]$

$$
\frac{d \sigma_{\text {classical }}^{\text {high-energy }}}{d \Delta E}\left(v, v_{e}, \Delta E\right)=2 \pi a_{0}^{2} \frac{E_{0}^{2}}{\Delta E^{3} m_{e} v^{2}}\left(\frac{2 m_{e} v_{e}^{2}}{3}+\Delta E\right) .
$$

Substituting Eq.(5) into Eq.(3) and Eq.(4) gives

$$
\begin{gathered}
\sigma_{\text {classical }}^{\text {high-energy }}\left(v, I_{n l}, Z_{p}\right)=\frac{5}{3} B_{n l} \sigma^{B o h r}\left(v, I_{n l}, Z_{p}\right), \\
B_{n l} \equiv \frac{3}{5}\left(\frac{2 K_{n l}}{3 I_{n l}}+1\right)
\end{gathered}
$$

where $\sigma^{B o h r}$ is given by Eq.(2), and $K_{n l} \equiv<m_{e} v_{e}^{2} / 2>_{n l}$ is the average orbital electron kinetic energy. For hydrogen-like electron orbitals, the average electron kinetic energy is equal to the ionization potential $K_{n l}=I_{n l}$ [23], and $B_{n l}=1$. The $B_{n l}$ factors are introduced to account for the difference in the electron velocity distribution functions (EVDF) from the EVDF of the hydrogen-like electron orbitals. The data for $K_{n l}$ are calculated for many atoms in Ref. [28]. For example, the average kinetic energy for the helium atom is $K_{n l} \equiv<m_{e} v_{e}^{2} / 2>=$ $1.43 E_{0}$, whereas $I_{n l}=0.91 E_{0}$, and therefore $B_{H e}=1.22$. That is the reason that accounting for the finite orbital electron velocity gives a cross section which is $5 / 3$ times larger than the Bohr formula in Eq.(2). This is a consequence of the fact that for an electron with nonzero velocity less energy transfer is required for ionization.

Classical mechanics gives the EVDF as a microcanonical ensemble, where

$$
f\left(v_{e}\right)=C v_{e}^{2} \int \delta\left(\frac{m_{e} v_{e}^{2}}{2}-\frac{Z_{T}}{r}+I_{n l}\right) r^{2} d r
$$


Here, $C$ is a normalization constant defined so that $\int f\left(v_{e}\right) d v_{e}=1$, and $\delta(\ldots)$ denotes the Dirac delta-function. Interestingly, the EVDF for a one-electron ion is identical in both the quantum-mechanical and classical calculations [23] with

$$
f\left(v_{e}\right)=\frac{32 v_{n l}^{5}}{\pi} \frac{v_{e}^{2}}{\left[v_{e}^{2}+v_{n l}^{2}\right]^{4}}
$$

where $v_{n l}$ is the scale of electron orbital velocity

$$
v_{n l}=v_{0} \sqrt{2 I_{n l} / E_{0}}
$$

Substituting the general differential cross section $d \sigma / d \Delta E\left(v_{e}, v, \Delta E\right)$ from Eq.(A3) of Appendix A and the EVDF in Eq.(8) into Eq.(3) yields

$$
\sigma^{G G V}\left(v, I_{n l}, Z_{p}\right)=\pi a_{0}^{2} Z_{p}^{2} \frac{E_{0}^{2}}{I_{n l}^{2}} G^{G G V}\left(\frac{v}{v_{n l}}\right) .
$$

Here, the scaling function $G^{G G V}(x)$ is given by Eq.(C3) in Appendix A, using the tabulation of the function $G(x)$ presented in Ref.[29] for $x>1$, and in Ref.[30] for $x<1$. The notation GGV stands for the classical trajectory calculation in Eq.(C3) due to Gerjuoy [27] using the fit of Garcia and Vriens [29].

\section{Bethe's treatment}

The classical calculations underestimate the cross sections for very high projectile velocities $v>>v_{n l}$. The scattering angle of the projectile due to collision with the target atom is of order $\theta_{c}=\Delta p / M v$, where $\Delta p$ is the momentum transfer in the collision, and $M$ is the mass of the projectile particle. The minimum energy transfer from the projectile is determined by the ionization potential, with $\Delta E=v \Delta p>I_{n l}$, and $\Delta p>\Delta p_{\min } \equiv I_{n l} / v$. Here, we use the fact that the momentum transfer $\Delta p$ is predominantly in the direction perpendicular to the projectile velocity. The projectile particle with wave vector $k=M v / \hbar$ undergoes diffraction on the object of the target atomic size $a_{n l}$ with the diffraction angle of order $\theta_{d}=1 /\left(k a_{n l}\right)=\hbar /\left(M v a_{n l}\right)$ [24]. At large projectile velocities $v>>v_{n l}$, it follows that $\Delta p_{\min } \equiv I_{n l} / v<<\hbar / a_{n l}$, because $v_{n l}=I_{n l} a_{n l} / \hbar$ for hydrogen-like electron orbitals. And for small $\Delta p \sim \Delta p_{\min }$, it follows that $\theta_{c}=\Delta p / M v<<\theta_{d}=\hbar /\left(M v a_{n l}\right)$. Therefore, the collision can not be described by classical mechanics. 
Bethe made use of the Born approximation of quantum mechanics to calculate cross sections [31] (see Appendix B for details). This yields for $v>>v_{n l}$

$$
\sigma^{\text {Bethe }}=\sigma^{\text {Bohr }}\left(v, I_{n l}, Z_{p}\right)\left[0.566 \ln \left(\frac{v}{v_{n l}}\right)+1.261\right] .
$$

If the projectile speed is much larger than the electron orbital velocity $v>>v_{n l}$, the logarithmic term on the right-hand side of Eq.(11) contributes substantially to the cross section, and as a result the quantum mechanical calculation in Eq.(11) gives a larger cross section than the classical trajectory treatment in Eq.(6). The quantum mechanical cross section is larger than the classical trajectory cross section due to the contribution of large impact parameters $(\rho)$ to the quantum-mechanical cross section, where the ionization is forbidden in classical mechanics because the energy transfer calculated by classical mechanics is less than the ionization potential $\left[\Delta E=v \Delta p_{c}(\rho)<I_{n l}\right.$, where $\Delta p_{c}$ is the momentum transfer given by classical mechanics in Eq.(1)]. However, ionization is possible due to diffraction in quantum mechanics [32]. Moreover, integration over these large impact parameters where the ionization is forbidden in classical mechanics, contributes considerably to the total ionization cross section (see Appendix B for further details).

\section{Gryzinski's treatment}

Gryzinski attempted to obtain the ionization cross sections using only classical mechanics similarly to Gerjuoy. But, in order to match the asymptotic behavior of the Bethe formula in Eq.(11) at large projectile velocities, Gryzinski assumed an artificial electron velocity distribution function (EVDF) instead of the correct EVDF in Eq.(8) [20], i.e.,

$$
f^{G r y z}\left(v_{e}\right)=\frac{1}{v_{n l}}\left(\frac{v_{n l}}{v_{e}}\right)^{3} \exp \left(-\frac{v_{n l}}{v_{e}}\right) .
$$

The ionization cross section was calculated by averaging the Rutherford cross section over all possible electron velocities, similar to the Gerjuoy calculation in Eq.(3), but was less accurate for small velocities $v<v_{n l}$. The effect of using the EVDF in Eq. (12) is to populate the EVDF tail with a much larger fraction of high-energy electrons with $v_{e}>>v_{n l}$, which gives $f^{G r y z}\left(v_{e}\right) \sim v_{e}^{-3}$ instead of $f\left(v_{e}\right) \sim v_{e}^{-6}$ for the correct EVDF in Eq.(8). As a result, the average electron kinetic energy $<m_{e} v_{e}^{2} / 2>$ diverges, which leads to a considerable enhancement of the ionization cross section at high projectile velocities. For 
$v>>v_{n l}$, Gerjuoy's calculation of the differential cross section $d \sigma / d \Delta E\left(v_{e}, v, \Delta E\right)$ of energy transfer $\Delta E$ is similar to Gryzinski's. Therefore, we can substitute Eq.(12) into Eqs.(5) and (4). Because in the limit $v>>v_{n l}$ the ionization cross section is proportional to the average electron kinetic energy $<m_{e} v_{e}^{2} / 2>$ [Eq.(6)], and the average kinetic energy diverges, it follows that a small population of high-speed electrons contributes considerably to the cross section. Using the general expression for $d \sigma / d \Delta E\left(v_{e}, v, \Delta E\right)$ avoids singularity and yields the logarithmic term in the ionization cross section similar to the Bethe formula in Eq.(11). After a number of additional simplifications and assumptions, Gryzinski suggested an approximation for the cross section in the form given by Eq.(10) with [20]

$$
\sigma^{G r y z}\left(v, I_{n l}, Z_{p}\right)=\pi a_{0}^{2} Z_{p}^{2} \frac{E_{0}^{2}}{I_{n l}^{2}} G^{G r y z}\left(\frac{v}{v_{n l}}\right) .
$$

Here, the function $G^{G r y z}(x)$ is specified by Eq.(C6) of Appendix C. In Eq.(13), the function $G^{G r y z}(x)$ has the following limit

$$
G^{G r y z}(x) \rightarrow[1+0.667 \ln (2.7+x)] / x^{2} \text { as } x \rightarrow \infty,
$$

which is close to Bethe's result in Eq.(11),

$$
G^{\text {Bethe }}(x) \rightarrow[1.261+0.566 \ln (x)] / x^{2} \text { as } x \rightarrow \infty .
$$

For $10<x<40$, it follows that

$$
G^{\text {Gryz }}(x) / G^{\text {Bethe }}(x) \simeq 1.04 .
$$

Therefore, the Gryzinski formula can be viewed as a fit to the Bethe formula at large velocities $v>>v_{n l}$ with some rather arbitrary continuation to small velocities $v<<v_{n l}$.

Figure 1 shows the experimental data for the cross section for ionizing collisions of fully stripped ions colliding with a hydrogen atom,

$$
X^{q+}+H(1 s) \rightarrow X^{q+}+H^{+}+e,
$$

where $X^{q+}$ denotes fully stripped ions of $H, H e, L i, C$ atoms, and (1s) symbolizes the ground state of a hydrogen atom. The experimental data for $\mathrm{H}^{+}$ions were taken from [33] (note that authors of this reference concluded that the previous measurements of the cross sections were inaccurate); from [34] for $\mathrm{He}^{+2}, \mathrm{C}^{+6}$ ions ; and from [35] for $\mathrm{Li}^{+3}$ ions. 


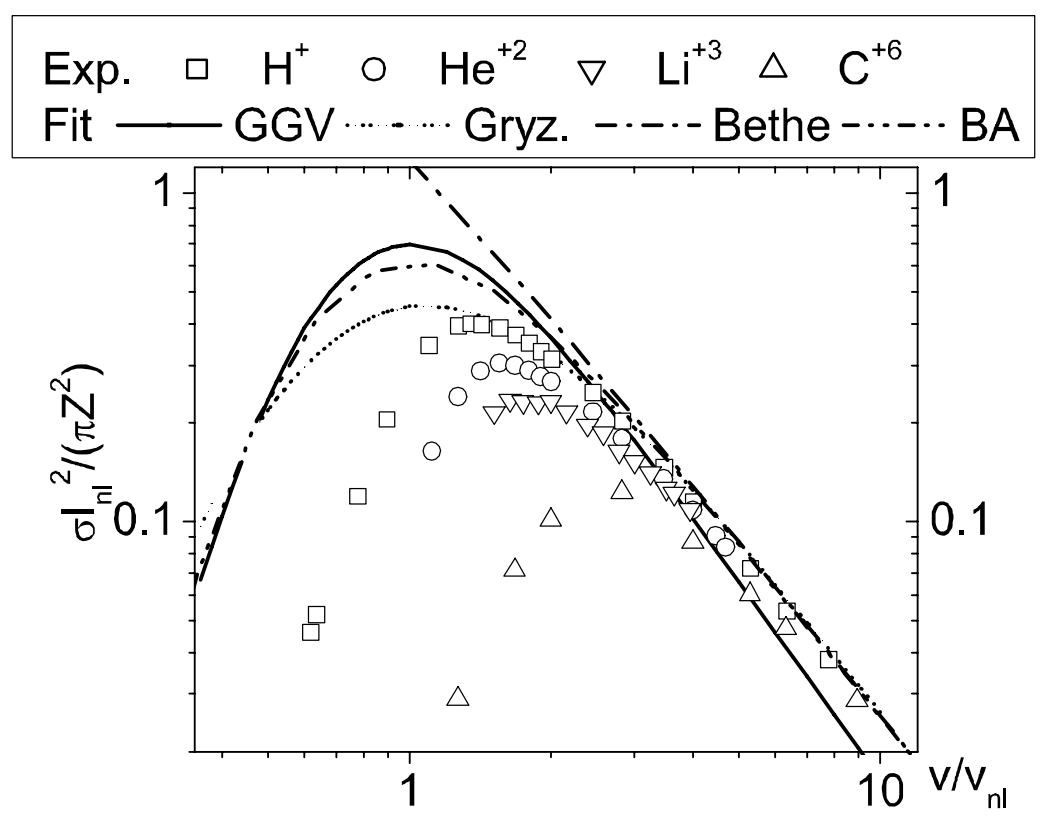

FIG. 1: Ionization cross sections of hydrogen by fully stripped ions showing both experimental data and theoretical fits. GGV stands for the classical calculation in Eq.(C3) due to Gerjuoy using the fit of Garcia and Vriens. Gryz denotes the Gryzinski approximation in Eq.(C6). Bethe stands for Bethe's quantum-mechanical calculation in the Born approximation, limited to $v>v_{n l}$ in Eq.(11). Finally, BA denotes the Born approximation in the general case in Eq.(21). All values are in atomic units. For hydrogen, the ionization potential $I_{n l}=1 / 2, v_{n l}=v_{0}=2.19 \cdot 10^{8} \mathrm{~cm} / \mathrm{s}$, and the cross section is normalized to $a_{0}^{2}=0.529^{2} \cdot 10^{-16} \mathrm{~cm}^{2}$.

From Fig. 1 it is evident that the Bethe formula describes well the cross sections for projectile velocities larger than the orbital velocities $v>>v_{n l}$. At large energies, the GGV formula underestimates the cross sections as discussed before, whereas Gryzinski's formula gives results close to the Bethe formula and the experimental data. Both, the GGV and Gryzinski formulas disagree with the experimental data at small energies.

\section{B. Behavior of cross sections at small projectile velocities $v<v_{n l}$}

The Bethe, GGV and Gryzinski's formulas fail at small velocities because they assume free electrons, neglecting the influence of the target atom potential on the electron motion 
during the collision. Apparently the assumption of free electron motion fails if the circulation period of the electron around the atom's nucleus is comparable with the interaction time of an ion with the electron. Let us now estimate the projectile velocities at which the electron circulation needs to be taken into account. The typical impact parameter leading to ionization is

$$
\rho_{\text {ioniz }} \simeq \sqrt{\frac{\sigma^{B o h r}}{\pi}}=\frac{2 a_{0} v_{0}^{2} Z_{p}}{v v_{n l}}
$$

and the interaction time is of order $\rho_{\text {ioniz }} / v$. The electron circulation time is $\tau_{n l} \simeq a_{n l} / v_{n l}$, where $v_{n l}$ is the electron orbital velocity, which scales as $v_{n l}=Z_{T} v_{0}$, and $a_{n l}$ is the ion radius $a_{n l}=a_{0} / Z_{T}[32]$. Therefore the condition $\tau_{n l}>\rho_{\text {ioniz }} / v$ holds for $v>v_{\max }$, where

$$
v_{\max }=v_{n l} \sqrt{2 Z_{p} / Z_{T}}
$$

Here, $Z_{p}$ is the charge of the fully stripped projectile and $Z_{T}$ is the nuclear charge of the target atom or ion. For velocities larger than $v_{\max }$, the ionization cross section decreases as the velocity increases [see Eq.(11)] due to the decreasing interaction time with an increase in velocity. On the other hand, for velocities less than $v_{\max }$, the collision becomes more adiabatic. The influence of the projectile is averaged out due to the slower motion of the projectile compared with the electron orbital velocity, and the ionization cross section decreases with decreasing projectile velocity. Thus, the cross section has a maximum at $v \simeq v_{\max }[$ Eq.(19)].

Note that if the projectile speed is comparable with or smaller than the electron orbital velocity $v<v_{n l}$, the Born approximation of quantum mechanical theory is not valid. Cumbersome quantum mechanical simulations are necessary for an exact calculation of the cross sections, as for example in Ref. [36]. Nevertheless for the case $2 Z_{p} \sim Z_{T}$ the maximum of the cross section calculated from the Born approximation is similar to the experimental results. To describe the behavior of the cross section near the maximum, the second-order correction in the parameter $v_{n l} / v$ has been calculated in [37], yielding the cross section in the form

$$
\sigma_{\text {mod }}^{\text {Bethe }}(\widetilde{v})=\frac{\pi a_{0}^{2}}{I_{n l}^{2}} \frac{Z_{p}^{2}}{\widetilde{v}^{2}}\left[0.566 \ln (\widetilde{v})+1.26-0.66 \frac{1}{\widetilde{v}^{2}}\right],
$$

where $\widetilde{v}=v / v_{n l}$. Equation(20) agrees with the exact calculation in the Born approximation [Eq.(B1)] as described in Appendix B (the agreement is within $10 \%$ for $\widetilde{v}>1.1$ ). We have developed the following fit for the cross section in the Born approximation in the general 
case,

$$
\sigma_{f i t}^{B A}(\widetilde{v})=\frac{\pi a_{0}^{2}}{I_{n l}^{2}} \frac{Z_{p}^{2}}{\widetilde{v}^{2}}\left[0.283 \ln \left(\widetilde{v}^{2}+1\right)+1.26\right] \exp \left[-\frac{1.95}{\widetilde{v}\left(1+1.2 \widetilde{v}^{2}\right)}\right] .
$$

Equation (21) agrees with the exact calculation [Eq.(B1)] within $2 \%$ for $\widetilde{v}>1$, and within $20 \%$ for $0.2<\widetilde{v}<1$.

Equation (21) was derived making use of the unperturbed atomic electron wave functions, which implicitly assumes that the projectile particle transfers momentum to the electron and departs to large distances, where it does not affect the electron to be ionized. The wave function can therefore be described as a continuous spectrum of the atomic electron, not affected by the projectile.

This assumption breaks down at low projectile velocities when the projectile velocity is comparable with the electron orbital velocity. Indeed, the electron kinetic energy in the frame of the projectile is of order $m_{e} v^{2} / 2$ and the potential energy $Z_{p} e^{2} / \rho_{\text {ioniz }}$, where $\rho_{\text {ioniz }}$ is the impact parameter leading to ionization, given by Eq.(18). Substituting $\rho_{\text {ioniz }}$ from Eq.(18) into electron potential energy $Z_{p} e^{2} / \rho_{\text {ioniz }}$ gives that potential energy is larger than kinetic energy if

$$
v<v_{n l} .
$$

Therefore, under the condition in Eq.(22), an electron can be effectively captured by the projectile after the collision instead of leading to ionization. As a result, the ionization cross section is small compared with the charge exchange cross section at low projectile velocities. The assumption of the unperturbed electron wave function results in grossly overestimated ionization cross sections as can be seen in Fig.1.

The ionization cross sections are also difficult to measure at small projectile energies, because careful separation between the large charge exchange cross section and the small ionization cross section is necessary for the correct measurement [33]. Therefore, early measurements of the ionization cross section at small velocities were not always accurate $[16,33]$.

\section{Gillespie's treatment}

To account for the difference between the Born approximation results and the experimental data for $v<v_{\max }$, Gillespie proposed to fit the cross sections to the following function 
$[22]$

$$
\sigma^{G i l l}(v)=\exp \left[-\lambda_{n l}\left(\frac{v_{0} \sqrt{Z_{p}}}{v}\right)^{2}\right] \sigma_{\text {mod }}^{B A}(v) .
$$

Here, $\lambda_{n l}$ is a constant, which characterizes the ionized atom or ion (for example, for the ground state of $\left.H, \lambda_{n l}=0.76\right)$, and $\sigma_{m o d}^{B A}$ is the cross section in the Born approximation in the form of Eq.(20). Gillespie's Eq.(23) proved to fit very well existing experimental cross sections for hydrogen atom ionization by $\mathrm{H}^{+}, \mathrm{He}^{+2}, \mathrm{Li}^{+2}, \mathrm{Li}^{+3}, \mathrm{C}^{+4}, \mathrm{~N}^{+5}, \mathrm{~N}^{+4}, \mathrm{O}^{+5}$ ions,

and less well for $H e$ and $H$ molecules with the same ions [22]. Because $\sigma_{\text {mod }}^{B A}(v)$ becomes negative for $v<0.7$, Gillespie's Eq.(23) can not be applied to these low projectile velocities. In principle, the general fit $\sigma_{\text {fit }}^{B A}$ in Eq.(21) can be used instead of $\sigma_{\text {mod }}^{B A}$ in Eq.(20). However, because the two formulas differ considerably in the range of interest, $0.7<v<1$, the fitting coefficients $\lambda_{n l}$ have to be updated for use with $\sigma_{f i t}^{B A}$.

Although Gillespie's fit proved to be very useful, there are a number of reasons to look for another fit. Gryzinski's Eq.(C6) is frequently used, because it requires only knowledge of one function for calculations of cross sections, notwithstanding the fact that it overestimates the cross sections at low energies.

\section{Olson's treatment}

For $v \lesssim v_{n l}$, a universal curve is expected if both the cross sections and the square of impact velocity are divided by $Z_{p}$ [41]. This scaling was established for the total electron loss cross section $\sigma^{e l}$, which includes both the charge exchange cross section $\sigma^{c e}$ and the ionization cross section. Based on the results of classical trajectory Monte Carlo (CTMC) calculations, Olson proposed the following fit [42],

$$
\sigma^{e l}\left(v, Z_{p}\right)=Z_{p} A_{n l} \pi a_{0}^{2} f^{\text {Olson }}\left(\frac{v}{v_{0} \gamma_{n l} \sqrt{Z_{p}}}\right)
$$

where $f(x)$ describes the scaled cross sections

$$
f^{\text {Olson }}(x)=\frac{1}{x^{2}}\left[1-\exp \left(-x^{2}\right)\right]
$$

Here, $\gamma_{n l}$ and $A_{n l}$ are constants, for example, $\gamma_{H}=\sqrt{5 / 4}=1.12$ and $A_{H}=16 / 3$ for atomic hydrogen, and $\gamma_{H e}=1.44$ and $A_{h e}=3.57$ for helium. The scaling in Eq.(24) was also demonstrated analytically by Janev [43]. For $v<<v_{0} \sqrt{Z_{p}}, \sigma^{e l}$ is dominated by 
charge exchange, $\sigma^{c e} \approx \sigma^{e l}$, and Eq.(24) gives a constant cross section for charge exchange, $\sigma^{c e} \approx \sigma^{e l}=16 \pi Z_{p} / 3 a_{0}^{2}$. For $v>>v_{0} \sqrt{Z_{p}}, \sigma^{e l}$ is dominated by the ionization cross section, and $\sigma^{c e} \approx \sigma_{\text {classical }}^{\text {high-energy }}$ [Eq.(6)]. Note that the scaling in Eq.(24) does not reproduce the logarithmic term in the Bethe formula [Eq.(11)] for $v>>v_{0} \sqrt{Z_{p}}$ because it is based on classical trajectory calculations. To make Eq. (24) agree with Eq.(6), the coefficients $\gamma_{n l}$ should be proportional to $\sqrt{I_{n l}}$. For example, the ionization potential for hydrogen is $I_{H}=$ $13.6 \mathrm{eV}$, and for helium $I_{H e}=24.6 \mathrm{eV}$. The ratio of $\gamma_{H}=1.12$ to $\gamma_{H e}=1.44$ differs from $\sqrt{I_{H}} / \sqrt{I_{H e}}$ by only five percent, i.e., $\gamma_{H} / \sqrt{I_{H}} /\left(\gamma_{H e} / \sqrt{I_{H e}}\right)=1.05$. Therefore, as was shown by Janev [43], the scaling in Eq.(24) can be rewritten in a form similar to Eq.(10) by normalizing the velocity to $v_{n l}$, Eq.(9), i.e.,

$$
\sigma^{e l}\left(v, I_{n l}, Z_{p}\right)=\pi a_{0}^{2} Z_{p} N_{n l} \frac{E_{0}^{2}}{I_{n l}^{2}} B_{n l} G^{e l}\left(\frac{v}{v_{n l} \sqrt{Z_{p}}}\right)
$$

where

$$
G^{e l}(x)=\frac{4}{3} f^{\text {Olson }}\left(x / \gamma_{H}\right)
$$

Here, $N_{n l}$ is the number of electrons in the orbital $n l$, and the $B_{n l}$ factors Eq.(7) are introduced to account for the difference of the orbital electron velocity distribution functions with the hydrogen-like EVDF function in Eq.(8). By construction, Eq.(25) coincides with Eq.(6) in the limit $v>>v_{n l} \sqrt{Z_{p}}$.

Because the scaling in Eq.(24) is based on classical trajectory calculations, it is valid only for intermediate velocities where the underbarrier transitions allowed in the quantum mechanical calculations do not contribute significantly (see Appendix B for details). Experimental data [35, 43] confirm the scaling in Eq. $(24)$ for $1.2<v /\left(v_{n l} \sqrt{Z_{p}}\right)<3$, or equivalently, for the projectile energy in the range $E=30-200 \times Z_{p} I_{n l} / I_{H}$ in units of $\mathrm{keV} / \mathrm{amu}$.

A similar scaling to Eq.(24) was derived in Ref.[50] based on quantum mechanical calculations making use of the quasi-classical approach developed originally by Keldysh for multi-photon ionization of atoms in a strong electromagnetic field. These calculations give scaling similar to Eq.(24), but with a different function $f(x)$ given in [50]. The quantum mechanical calculation results for the charge exchange cross section in Ref. [50] are a factor of 3 larger than Olson's cross section in Eq.(24) for $v /\left(v_{0} \sqrt{Z_{p}}\right)<0.2$.

Direct application of the scaling in Eq.(25) for the ionization cross section instead of the total electron removal cross section does not produce a single scaled function [see Fig.2 for 


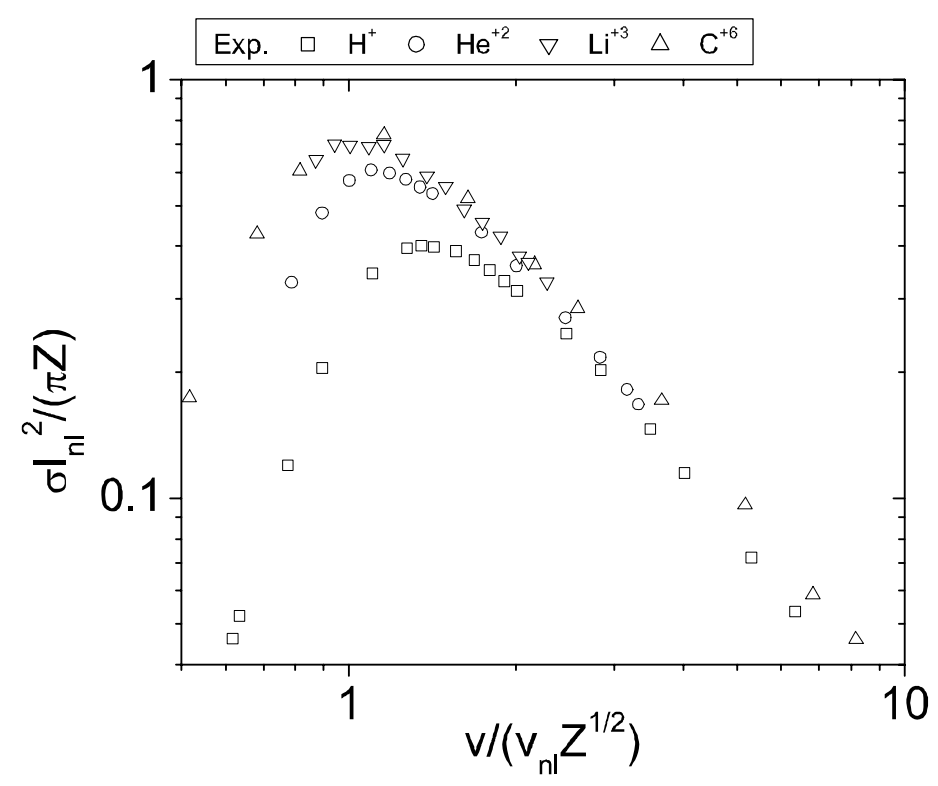

FIG. 2: Ionization cross sections of hydrogen by fully stripped ions. The scaled experimental data are from Fig.1. Note that the data do not merge into a single curve.

hydrogen and Fig. 4.(b) for helium]. Furthermore, the data are considerably scattered near the maxima of the cross sections.

\section{NEW FIT FORMULA FOR THE IONIZATION CROSS SECTION}

Analysis of the experimental data in Fig.1 shows that the maxima of the experimentally measured cross sections occur at $\sqrt{Z_{p}+1}$, not at $\sqrt{Z_{p}}$ as would be the case according to Olson's scaling in Eq.(24). Therefore, it is natural to plot cross sections as a function of the normalized velocity $v /\left(v_{n l} \sqrt{Z_{p}+1}\right)$. Note that at large velocities, according to Eq.(6) $\sigma \sim Z_{p}^{2} / v^{2}$. Therefore, making use of the normalized velocity $v /\left(v_{n l} \sqrt{Z_{p}+1}\right)$ requires normalization of the cross sections according to $\sigma /\left[Z_{p}^{2} /\left(Z_{p}+1\right)\right]$. As a consequence, instead of Eq.(25), we propose the following scaling

$$
\sigma^{i o n}\left(v, I_{n l}, Z_{p}\right)=\pi a_{0}^{2} \frac{Z_{p}^{2}}{\left(Z_{p}+1\right)} N_{n l} \frac{E_{0}^{2}}{I_{n l}^{2}} G^{\text {new }}\left(\frac{v}{v_{n l} \sqrt{Z_{p}+1}}\right) .
$$


Resulting plots of the scaled cross sections are shown in Fig.3. Comparing Fig.2 and Fig.3(b) one can clearly see that all of the experimental data merge close to each other on the scaled plot based on Eq.(26).

The resulting universal function can be fitted with various functions, but the simplest fit was proposed by Rost and Pattard [45]. They showed that if both the cross section and the projectile velocity are normalized to the values of cross section and projectile velocity at the cross section maximum, then the scaled cross section $\sigma / \sigma_{\max }$ is well described by the fitting function

$$
\sigma(v)=\sigma_{\max } \frac{\exp \left(-v_{\max }^{2} / v^{2}+1\right)}{v^{2} / v_{\max }^{2}} .
$$

Here, $\sigma_{\max }$ is the maximum of the cross section, which occurs at velocity $v_{\max }$. For the present study (the case of the ionization cross section by the bare projectile), we predict that

$$
\begin{aligned}
\sigma_{\max } & =\pi a_{0}^{2} B_{n l} \frac{Z_{p}^{2}}{\left(Z_{p}+1\right)} \frac{E_{0}^{2}}{I_{n l}^{2}}, \\
v_{\max } & =v_{n l} \sqrt{Z_{p}+1},
\end{aligned}
$$

where the coefficients $B_{n l}$ depend only weakly on the projectile charge. From Fig.3 one can estimate $B_{n l}=0.8$ for the ionization of hydrogen by protons, while for ionization of hydrogen by bare nuclei of helium and lithium, we find $B_{n l}=0.93$. As can be seen from Fig.3, the function in Eq.(27) with $\sigma_{\max }$ and $v_{\max }$ defined in Eq.(28) describes well the cross sections at small and intermediate energies, but underestimates the cross section at high energies. The reason is that the function in Eq.(27) does not reproduce the logarithmic term in the Bethe formula in Eq.(11). To improve the agreement with the experimental data and the Bethe formula we propose a new scaling for the fitting function in Eq.(26) defined by

$$
G^{\text {new }}(x)=\frac{\exp \left(-1 / x^{2}\right)}{x^{2}}\left[1.26+0.283 \ln \left(2 x^{2}+25\right)\right] .
$$

At large $x>>1$, Eq.(30) approaches the Bethe formula in Eq.(15), and at small $x<1$, Eq.(30) approaches the result in Eq.(27). The function $G^{\text {new }}(x)$ has a maximum at $x \simeq 1$, with $G^{\text {new }}(1) \simeq 0.86$. Because 0.86 is in between the maxima of the scaled cross section of hydrogen by protons $\left(B_{n l}=0.8\right)$ and the cross section for ionization of hydrogen by bare nuclei of helium and lithium $\left(B_{n l}=0.93\right)$, we did not incorporate the coefficients $B_{n l}$ in Eq.(30). This gives it a general form and introduces small errors of less than $8 \%$. 

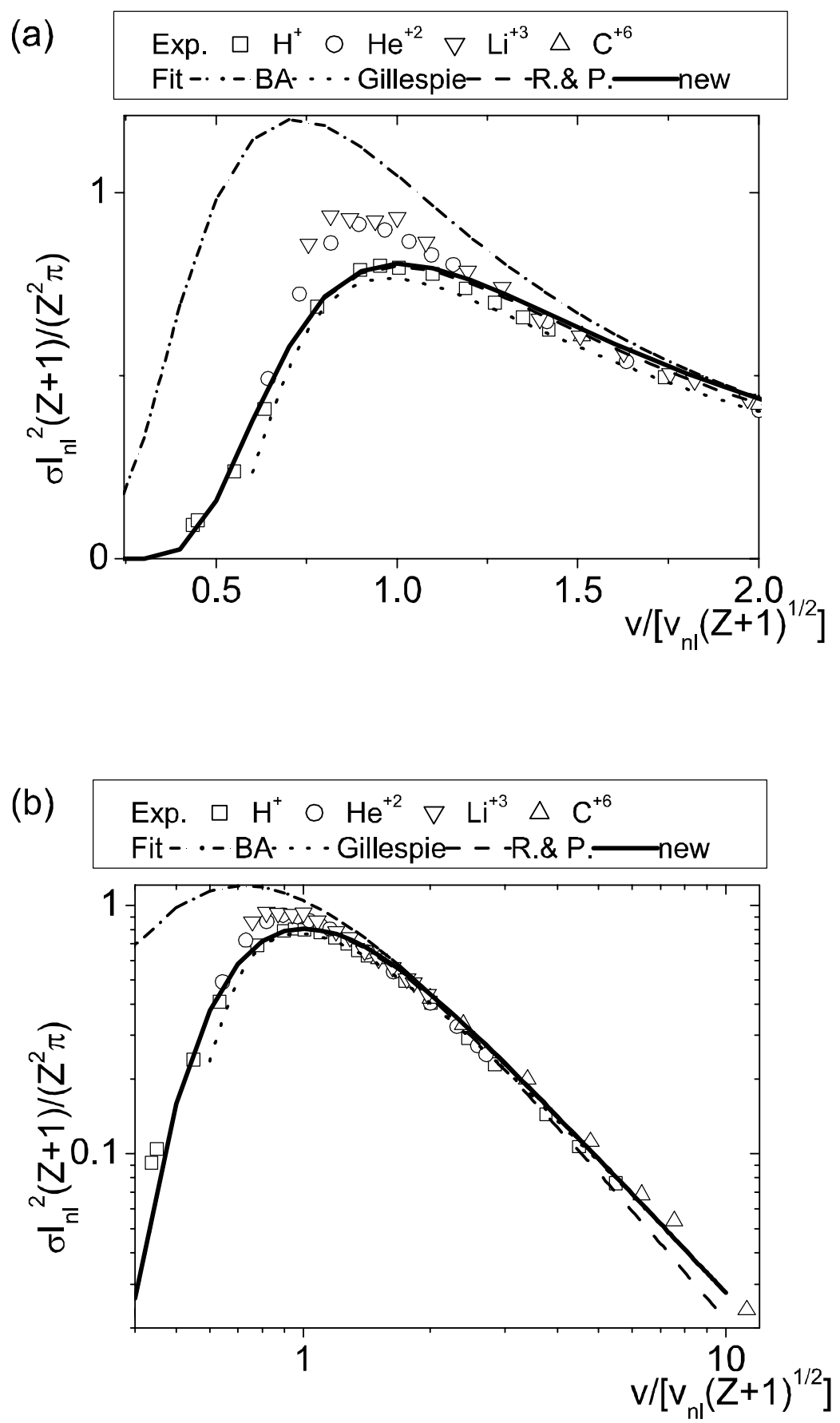

FIG. 3: Ionization cross sections of hydrogen by fully stripped ions showing the scaled experimental data and the theoretical fits. BA denotes the Born approximation [Eq.(21)]. Gillespie denotes Gillespie's fit according to Eq.(23). R.\&P. symbolizes the fit proposed by Rost and Pattard [45] in Eq.(27). "New" denotes the new fit given by Eq.( 30). The information in Fig. 3(b) is the same as in Fig.3(a) but presented as a log-log plot for better viewing of the cross sections for low and high projectile velocities. 

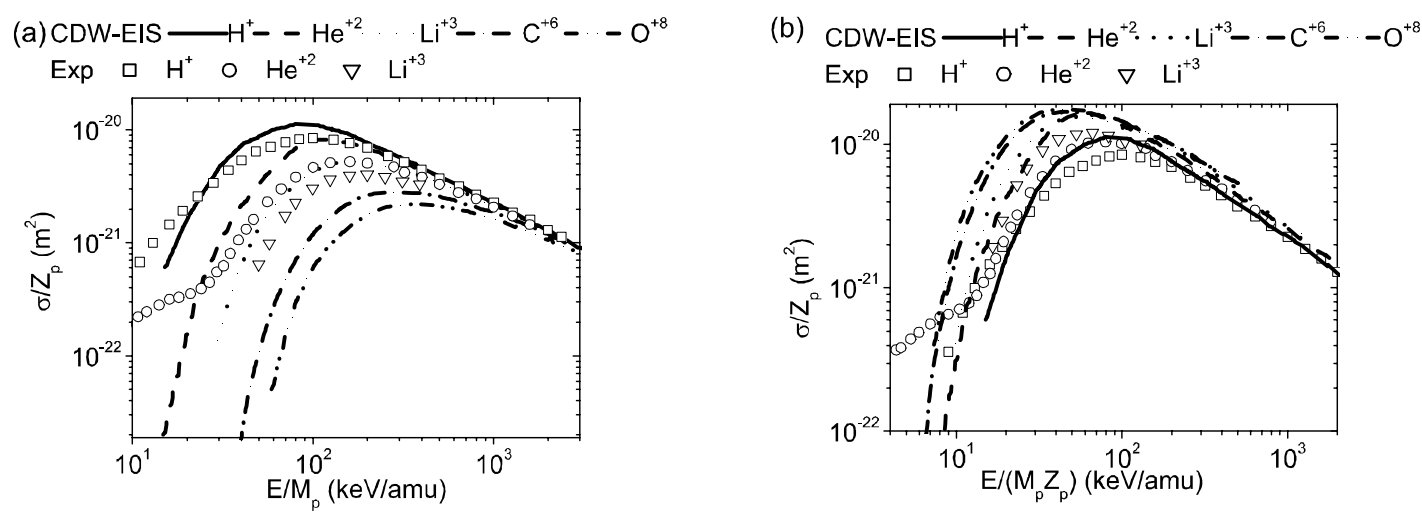

(c) CDW-EIS $-\mathrm{H}^{+}--\mathrm{He}^{+2} \cdots \mathrm{Li}^{+3}--\mathrm{C}^{+6}-\cdots-\mathrm{O}^{+8}$
$\operatorname{Exp} \square \mathrm{H}^{+} \circ \quad \mathrm{He}^{+2} \nabla \mathrm{Li}^{+3}$

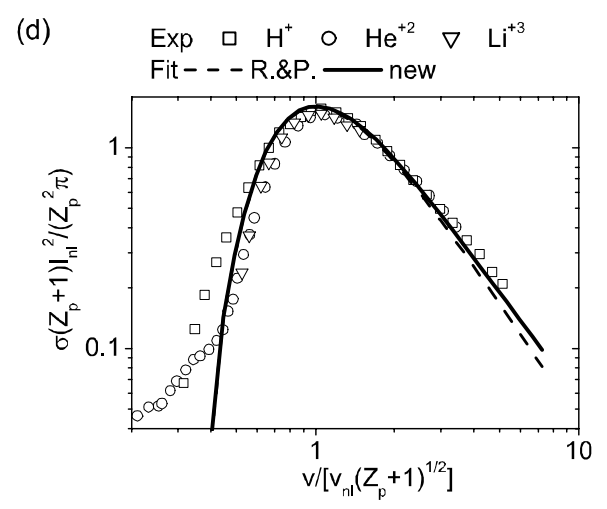

FIG. 4: Ionization cross sections of helium by fully stripped ions. The experimental data is from Refs. [46] and [47], and the theoretical calculations from Ref. [48]. Showing in the figures are (a) the raw data; (b) the scaled data from Fig.4a, making use of Eq.(25); (c) the scaled data making use of Eq.(26); and (d) the experimental data only scaled using Eq.(26), and comparing with the fit functions. The notation R.\&P. denotes Eq.( 27), and "new" denotes Eq.(30).

We have applied the new fit in Eqs.(26) and (30) to the ionization cross section of helium, shown in Fig.4a. The symbols denote the experimental data from Refs. [46] and [47], and the lines correspond to the continuum-distorted-wave-eikonal initial state (CDW-EIS) theory [48], which is a generalization of the Born approximation. The CDW-EIS theory accounts for the distortion of the electron wave function by the projectile. From Fig.4a one can see that the CDW-EIS theory overestimates the cross section near the maximum, and underestimates the cross section at small energies.

Direct application of the scaling formula in Eq.(25) to the ionization of helium does not produce similar good results to the hydrogen case [see Fig. 4(b)]. But after applying the 
new scaling in Eq.(26), all of the experimental and theoretical results merge close together on the scaled plot, as is clearly evident in Fig.4(c). Moreover, if we plot the cross sections as a function of velocity normalized to the orbital velocity $v_{n l}$ estimated from the ionization potential of helium $\left(I_{H e}=24.6 \mathrm{eV}\right)$ making use of Eq.(9), the cross section is given by the same scaling as in Eq.(26) with the same function as in Eq.(30), as evident from Fig.4(d). (The number of electrons in the helium atom is $N_{n l}=2$, and therefore the scaled cross section is twice that of hydrogen.) From Fig.4(d) it is clear that the new proposed fit in Eq.(26) using the function in Eq.(30) gives very good results for both hydrogen and helium.

Further verification of the new scaling is difficult because reliable experimental data and numerical simulations for a broad range of projectile velocities are absent for other atoms. The discrepancy between the new fit and the helium data at very small velocities is discussed in the next section.

\section{THEORETICAL JUSTIFICATION FOR THE NEW FIT FORMULA FOR IONIZATION CROSS SECTION}

In this section we discuss the theoretical foundations for the new fit to the ionization cross section given by Eq.(26) and Eq.(30). We start with an analysis of high projectile velocities.

\section{A. Behavior of cross sections at large projectile velocities $v>v_{n l}$}

In the region of high projectile velocities the new fit predicts the ionization cross section

$$
\sigma_{f i t}^{\text {high-energy }}(v)=4 \pi a_{0}^{2} \frac{v_{0}^{4}}{v_{n l}^{2}} \frac{Z_{p}^{2}}{v^{2}}\left[0.566 \ln \left(\frac{v}{v_{n l} \sqrt{\left(Z_{p}+1\right) / 2}}\right)+1.26\right],
$$

which differs from the Bethe formula in Eq.(11). [The factor $\sqrt{\left(Z_{p}+1\right) / 2}$ appears in the denominator under the logarithm in the first term on the right hand side of Eq.(31).] We claim that incorporating this factor gives a better cross section estimate than the Bethe formula. A comparison of the existing experimental data with the Bethe formula in Eq.(11) and the fit formula in Eq.(31) is shown in Fig.5. The experimentally estimated uncertainty of $5.5 \%$ [35] is shown by the error bar. The region of validity of the Born approximation 


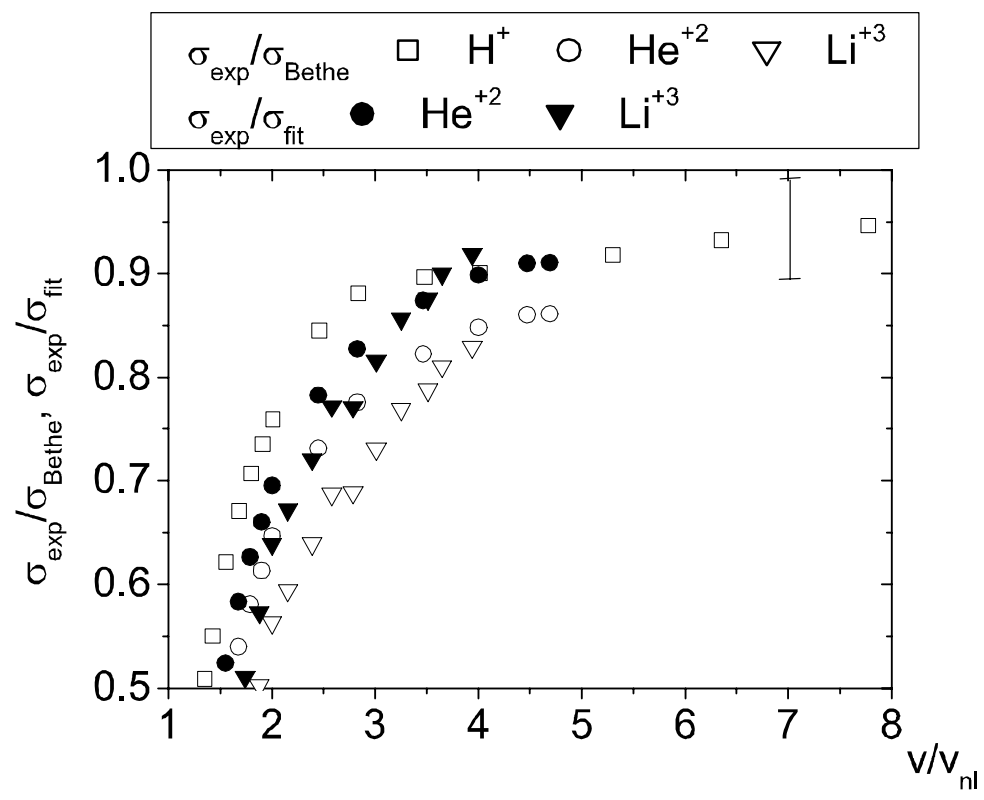

FIG. 5: Ratio of ionization cross sections of hydrogen by fully stripped ions to the Bethe formula in Eq.(11) and the fit formula in Eq.(31) at high velocities. The experimentally estimated uncertainty of $5.5 \%[35]$ is shown by the error bar.

and, hence, the Bethe formula is [23, 24]

$$
v>\max \left(2 Z_{p} v_{0}, v_{n l}\right)
$$

The first condition in Eq.(32) assures that the projectile potential is taken into account in the Born approximation; the second condition allows use of the unperturbed atomic wave function. Unfortunately the experimental data exists in the region in Eq.(32) only for the ionization of hydrogen by protons. Figure 5 shows that the Bethe formula describes the experimental data for ionization of hydrogen by protons within the error bar only for $v>6 v_{0}$. Application of the fit formula instead of the Bethe formula reduces discrepancy with the data. To further discriminate between the fit formula and the Bethe formula, new systematic experiments or exact quantum mechanical calculations are necessary for large $Z_{p}$ and high velocities $v>Z_{p} v_{0}$.

The applicability of the Bethe formula is limited by the validity of the Born approximation. One of the easiest ways to correct it was suggested in Ref.[50]. Firstly, the Born approximation is considered, making use of a classical trajectory for the projectile and a 
quantum mechanical description in the Born approximation for the electron. In this approximation, the probability of ionization or excitation is a function of the impact parameter $\rho$. Here, for brevity, we shall consider only ionization of the hydrogen atom. The projectile particle interacts with the atomic electron with a potential energy $V\left(\mathbf{R}, \mathbf{r}_{e}\right)=-Z_{p} e^{2} /\left|\mathbf{R}-\mathbf{r}_{e}\right|$, where $\mathbf{R}(t)=\boldsymbol{\rho}+\mathbf{v} t$ is the classical trajectory of the projectile particle, and $\mathbf{r}_{e}$ describes the position of the electron relative to the nucleus of the atom. For any impact parameter $\rho$, the probability of ionization is given by the square of the transition amplitude

$$
P_{B A}(\rho)=\frac{1}{\hbar^{2}}\left|\int d \mathbf{r}_{e} \Psi_{i}\left(r_{e}\right)\left[\int d t e^{i \Delta E t / \hbar} V\left(\mathbf{R}, \mathbf{r}_{e}\right)\right] \Psi_{f}^{*}\left(r_{e}\right)\right|^{2} .
$$

Here, $\Delta E$ is the transferred energy in the transition, and $\Psi_{i}$ and $\Psi_{f}$ are the initial and final electron wave functions, respectively. It can be shown that the calculations of ion-atom ionization cross sections using the conventional Born approximation (outlined in Appendix B) and using the straight line classical projectile trajectory [Eq.(33)] are equivalent [32].

For large impact parameters $\rho>>a_{0}$, we can expand $V\left(\mathbf{R}, \mathbf{r}_{e}\right)$ in powers of $\mathbf{r}_{e n l} / R$ according to

$$
V\left(\mathbf{R}, \mathbf{r}_{e}\right)=Z_{p} e^{2}\left(-\frac{1}{R}+\frac{\mathbf{R} \cdot \mathbf{r}_{e}}{R^{3}}\right) .
$$

The first term does not contribute to the matrix element in Eq.(33) due to the orthogonality of the final and initial states. Substituting Eq.(34) into Eq.(33) and integrating in time yields [32]

$$
P_{B A}(\rho)=\left(\frac{2 Z_{p} v_{0}}{\rho v}\right)^{2}\left|\int d \mathbf{r}_{e} \Psi_{i}\left(r_{e}\right) \Psi_{f}^{*}\left(r_{e}\right)\left[\frac{\omega \rho}{v} x_{e} K_{1}\left(\frac{\omega \rho}{v}\right)+i z_{e} \frac{\omega \rho}{v} K_{0}\left(\frac{\omega \rho}{v}\right)\right]\right|^{2},
$$

where $\omega=\Delta E / \hbar$, and $K_{n}$ is the modified Bessel function. Expanding the Bessel functions for small and large arguments, or simply evaluating the integrand in Eq.(35) approximately, we can approximate

$$
\frac{\omega \rho}{v} K_{1}\left(\frac{\omega \rho}{v}\right)=\left\{\begin{array}{c}
1, \frac{\omega \rho}{v}<1 \\
0, \frac{\omega \rho}{v}>1
\end{array}\right\}
$$

and neglect the second term on the right hand side in Eq.(35), which is small compared with the first term. The probability of ionization vanishes for $\rho>\rho_{\max } \simeq v / \omega=2 a_{0} v / v_{0}$, corresponding to the adiabatic limit. For $\rho>\rho_{\max }$, the collision time $\rho_{\max } / v>a_{0} / v_{0}$ is much longer than the electron circulation time around the nucleus, and the collision is adiabatic. Consequently, the ionization probability is exponentially small for $\rho>2 a_{0} v / v_{0}$. 
The square of electron dipole matrix element averaged over all possible momenta of the ionized electron is [31]

$$
\sum_{f} \int d \mathbf{r}_{e}\left|\Psi_{i}\left(r_{e}\right) x_{e} \Psi_{f}^{*}\left(r_{e}\right)\right|^{2}=0.283 a_{0}^{2}
$$

Note that the sum over all final states including both ionization and excitation gives

$$
\sum_{f}<0\left|x_{e}\right| f><f\left|x_{e}\right| 0>=<0\left|x_{e}^{2}\right| 0>=\frac{1}{3}<0\left|r_{e}^{2}\right| 0>=a_{0}^{2} .
$$

In this sum, 0.717 corresponds to excitation, and 0.283 corresponds to ionization [31].

For large impact parameters the momentum transfer to the electron is small and we can neglect the electron kinetic energy of the ejected electron compared with the ionization potential. As a result, $\Delta E \approx I_{H}=E_{0} / 2$ and $\omega=v_{0} / 2 a_{0}$ (in atomic units). Finally for $\rho>a_{0}$, the ionization probability is

$$
P_{B A}(\rho) \approx 0.283\left(\frac{2 a_{0} v_{0} Z_{p}}{\rho v}\right)^{2}\left\{\begin{array}{l}
1, \rho<2 a_{0} v / v_{0} \\
0, \rho>2 a_{0} v / v_{0}
\end{array}\right\}
$$

The ionization cross section is given by the integral

$$
\sigma=2 \pi \int_{0}^{\infty} P_{B A}(\rho) \rho d \rho
$$

For $\rho>a_{0}$, we can use Eq.(39) to estimate $P_{B A}(\rho)$. For $\rho<a_{0}$, the dipole approximation in Eq.(34) is not valid. To evaluate $P_{B A}(\rho)$ approximately for $\rho<a_{0}$, we can utilize the fact that $\int d t e^{i \Delta E t / \hbar} V\left(\mathbf{R}, \mathbf{r}_{e}\right)$ is a weak function of $\rho$ for $\rho<a_{0}$, and therefore $P_{B A}(\rho) \approx P_{B A}\left(a_{0}\right)$. Substituting $P_{B A}(\rho) \approx P_{B A}\left(a_{0}\right)$ for $\rho<a_{0}$, and $P_{B A}(\rho)$ from Eq.(39) for $\rho>a_{0}$, into Eq.(40) gives

$$
\sigma=8 \pi a_{0}^{2} \cdot 0.283 \frac{v_{0}^{2} Z_{p}^{2}}{v^{2}}\left[\frac{1}{2}+\ln \left(\frac{2 v}{v_{0}}\right)\right]
$$

The first term in Eq.(41) comes from contributions of impact parameters $\rho<a_{0}$, and the second term originates from contributions of large impact parameters $\rho>a_{0}$, respectively. Comparison with the exact result in the Born approximation in Eq.(11) shows that the contribution of impact parameters $\rho<a_{0}$ is underestimated, and $1 / 2$ should be replaced by 1.52. The above considerations are valid if the total probability of ionization and excitation $\left[P_{B A}^{t o t}(\rho)=\left(2 Z_{p} a_{0} v_{0} / \rho v\right)^{2}\right.$, for $\left.\rho>a_{0}\right]$ for the entire region of impact parameters is less than unity, which requires $2 Z_{p} v_{0} / v<1$. (Note that the total probability of ionization and excitation is about 4 times larger for ionization only.) 
For $2 Z_{p} v_{0} / v>1$, the total probability of the ionization and excitation $P_{B A}^{t o t}(\rho)$ calculated using the Born approximation is more than unity, $P_{B A}^{t o t}(\rho)>1$, for impact parameters $\rho<\rho_{\text {break }}=2 Z_{p} a_{0} v_{0} / v$, indicating the breakdown of the Born approximation [50]. Similar to the previous case, we can estimate the ionization probability $P_{B A}(\rho)$ from Eq.(39) for $\rho>$ $\rho_{\text {break }}>a_{0}$ and assume $P_{B A}(\rho) \approx P_{B A}\left(\rho_{\text {break }}\right)=0.283$ for $\rho<\rho_{\text {break }}$. These considerations result in a cross section estimate similar to the Bethe formula but with the logarithmic term in the form $\ln \left(\rho_{\max } / \rho_{\min }\right)=\ln \left(v^{2} / v_{0}^{2} Z_{p}\right)$, which gives

$$
\sigma=8 \pi a_{0}^{2} \cdot 0.283 \frac{v_{0}^{2} Z_{p}^{2}}{v^{2}}\left[\frac{1}{2}+\ln \left(\frac{v^{2}}{v_{0}^{2} Z_{p}}\right)\right] .
$$

This calculation results in a smaller cross section than the Bethe formula for $2 Z_{p} v_{0} / v>1$. Note that in the above analysis we have used unperturbed electron wave functions, which is valid only for $v>>v_{0}$.

The rigorous approaches to improve the Bethe formula are based on the eikonal approximation instead of the Born approximation [49]. The eikonal approximation is justified if $k a_{n l}>1$, where $k$ is the projectile particle wave vector $k=M v / \hbar$, and the projectile kinetic energy is large compared to the potential energy interaction with the target. For heavy projectile particles with mass much larger than the electron mass, these conditions are well satisfied. The ionization cross section in the eikonal approximation is given by [23]

$$
\sigma=2 \pi \int \frac{q d q}{k^{2}}|f(\mathbf{q})|^{2}
$$

where $f(\mathbf{q})$ is the amplitude of ionization with momentum transfer $\mathbf{q}$

$$
f(\mathbf{q})=\frac{k}{2 \pi i} \int \rho d \rho<\text { final }\left|\exp \left(\frac{i \int V d z}{\hbar}-i \mathbf{q} \cdot \boldsymbol{\rho}\right)\right| \text { initial }>\text {. }
$$

The eikonal approximation in Eqs.(43) and (44) accounts approximately for all orders of the perturbation series, whereas the Born approximation only make use of the first order. The calculations in the eikonal approximation yield a formula similar to Eq.(42) [51]. Note that the validity of the eikonal approximation in Eq.(44) is limited to $v>>v_{0}$, because the electron wave functions $\Psi_{i}$ and $\Psi_{f}$ are assumed to be unperturbed atomic functions. The influence of the projectile on the electron wave functions has to be taken into account for $v \lesssim v_{0}$. This is typically performed in the distorted wave approximation [16].

Therefore, the correction to the Born approximation in Eq.(42) and the eikonal approximation give a formula similar to Eq.(31) but with a factor $\alpha \sqrt{Z_{p}}$ ( $\alpha$ is a coefficient of order unity), instead of $\sqrt{\left(Z_{p}+1\right) / 2}$. At large velocities, both formulas give similar results. 


\section{B. Behavior of cross sections at small projectile velocities $v<v_{n l}$}

If the projectile velocity is small compared with the orbital velocity, the collision is adiabatic and the electron circulates many times around both nuclei. The electronic energy states need to be determined in such a quasimolecule as a function of the positions of both nuclei at a particular time. In both the quantum mechanical and the classical approaches, ionization is only possible if during the collision the initial and final electronic terms cross at some instant. In classical mechanics this corresponds to the so-called " $v / 2$ mechanism". In a collisional system comprised of two nuclei of equal charges (say ionization of hydrogen by a proton), an electron which is exactly in between the two nuclei experiences a very small electric field because the electric fields from both nuclei exactly cancel for all times at this point. The electron can "ride" this saddle point of the potential if its velocity is equal to one-half the velocity of the projectile. The collision dynamics is illustrated in Fig.6.

From Fig. 6 one can see that the electron is stranded in between the protons at $t=15 a_{0} / v_{0}$ and its velocity projection on the $\mathrm{x}$-axis is one-half of the projectile velocity. A small variation of the initial condition from $z=-1.606756 a_{0}$ (solid line) to $z=-1.606751 a_{0}$ (dotted line) completely changes the result of the collision. After the collision the electron stays near the first nucleus and does not become ionized. As a result, the probability of ionization is extremely small even though the projectile velocity is not small (for the conditions in Fig.6, $v=1 / 2$ in atomic units). The mechanism for ionization described above is also so-called T-promotion in quantum mechanical descriptions [53].

Another mechanism for ionization is attributed to the so-called S-promotion mechanism [53]. It is associated with the special type of trajectory of the electron in the field of two positive charges, shown in Fig.6(c). Figure 6(c) shows that an electron with particular initial conditions tends to spiral with a large number of turns enclosing a segment of the straight line joining the nuclei Fig.6(c) [54]. Such a trajectory is unstable - a small variation of initial conditions results in a completely different trajectory as shown in Fig.6(c). Analysis of the electron motion in the field of two positive charges, $Z_{T}$ and $Z_{P}$, which are separated by a distance $R$ is best described in elliptical coordinates

$$
\xi=\frac{r_{p}+r_{T}}{R}, \eta=\frac{r_{p}-r_{T}}{R},
$$

where $r_{p}$ and $r_{T}$ are the distances from the electron to the projectile and target nuclei, respectively. Making use of atomic units, the classical trajectory in terms of the variables $\xi$ 
(a)

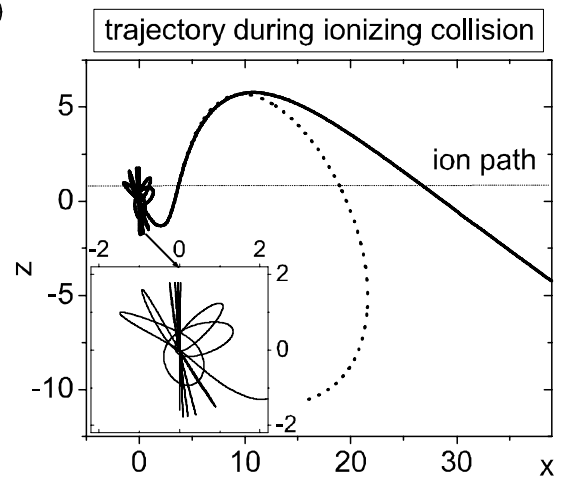

(c)

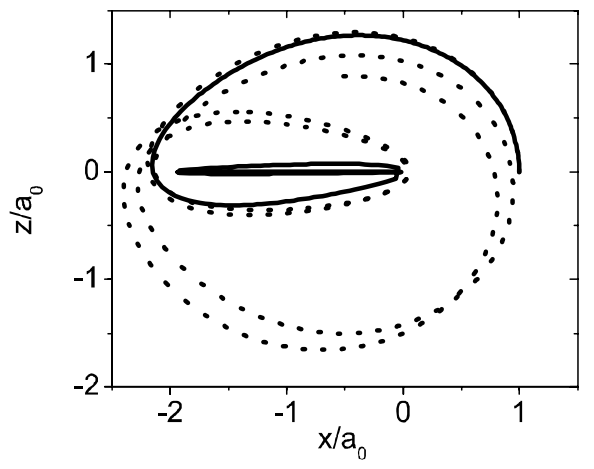

(b)

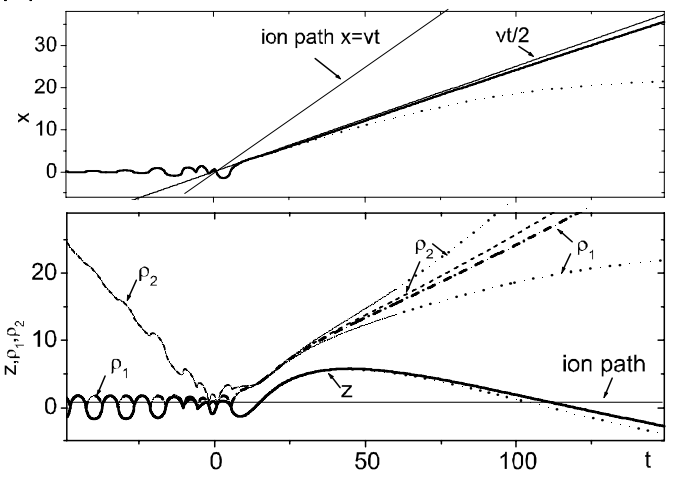

FIG. 6: The trajectory of a $v / 2$ collision is shown in Figs.6(a) and (b). The initial conditions correspond to a hydrogen atom with total energy $-1 / 2$, and at $t=-60 x=0=y, v_{x}=0=v_{y}$, $z=-1.606756$ (solid line) and $z=-1.606751$ (dotted line). The projectile moves along $z=1$ with velocity $1 / 2$. Atomic units are used: velocity is normalized to $v_{0}$; distance is normalized to $a_{0}$; and time is normalized to $a_{0} / v_{0}$. Figure $6(\mathrm{~b})$ shows the position $[x(t), z(t)]$ of the electron as a function of time, and the distance between the electron and the first $\left(\rho_{1}\right)$ and the second proton $\left(\rho_{2}\right)$ for the same conditions as in Fig.6(a). The trajectory of a $S$-promotion is shown in Figs.6(c) for fixed positive charges $(v \rightarrow 0)$. The initial conditions correspond to an internuclear separtion $2 a_{0}$ (in atomic units), initial position of the electron $z=0, x=1$; and initial velocity $v_{x}=0$, $v_{z}=1.155$ (solid line), and $v_{z}=1.165$ (dotted line).

and $\eta$ can be expressed as [54]

$$
\frac{d \xi}{d t}=\frac{4\left(\xi^{2}-1\right) P_{\xi}}{R^{2}\left(\xi^{2}-\eta^{2}\right)}, \frac{d \eta}{d t}=-\frac{4\left(\eta^{2}-1\right) P_{\eta}}{R^{2}\left(\xi^{2}-\eta^{2}\right)},
$$


where the canonical momentums $P_{\xi}$ and $P_{\eta}$ are

$$
\begin{aligned}
& P_{\xi}=\left(-\frac{1}{2} R^{2}|E|+\frac{\left(Z_{P}+Z_{T}\right) R \xi-\lambda}{\xi^{2}-1}-\frac{P_{\phi}^{2}}{\left(\xi^{2}-1\right)^{2}}\right)^{1 / 2} \\
& P_{\eta}=\left(-\frac{1}{2} R^{2}|E|+\frac{\left(Z_{P}-Z_{T}\right) R \eta+\lambda}{1-\eta^{2}}-\frac{P_{\phi}^{2}}{\left(1-\eta^{2}\right)^{2}}\right)^{1 / 2}
\end{aligned}
$$

Here $E<0$ is the total energy of the electron, $P_{\phi}=\xi \eta d \phi / d t$ is the rotational momentum around the straight line joining the nuclei, and $\lambda$ is the integral of motion (for stationary nuclei)

$$
\lambda=M^{2}-\frac{R^{2}}{4}\left(P_{\zeta}^{2}+\frac{P_{\phi}^{2}}{\zeta^{2}}\right)+R\left(Z_{P} \cos \theta_{P}+Z_{T} \cos \theta_{T}\right) .
$$

Here, $\zeta$ is the closest distance from the electron to the straight line joining the nuclei; $P_{\zeta}$ is the vector dot product of the electron momentum with the $\zeta$-axis; $M^{2}=(\mathbf{r} \times \mathbf{p})^{2}$ is the total rotational momentum; and $\theta_{P}$ and $\theta_{T}$ are the angles between $\mathbf{r}_{p}$ and $\mathbf{R}$, and $\mathbf{r}_{T}$ and $-\mathbf{R}$, respectively. Moreover, $\mathbf{r}_{p}$ is the radius vector from the projectile to the electron; $\mathbf{r}_{T}$ is the radius vector from the target nucleus to the electron; and $\mathbf{R}$ is the radius vector from the projectile to the target nucleus. The canonical momentum $P_{\xi}$ in Eq.(47) tends to infinity if $\xi \rightarrow 1$, preventing the electron from approaching a segment of the straight line joining the nuclei, $\xi=1$. In the special case

$$
\left(Z_{P}+Z_{T}\right) R=\lambda, P_{\phi}=0
$$

the singularity vanishes at the point $\xi=1$ in Eq.(47). As a result, for initial conditions satisfying the condition in Eq.(50), $P_{\xi}$ is finite for $\xi=1$. From Eq.(46), $\xi$ approach unity exponentially with time - the limiting electron trajectory lies on the internuclear axis - as shown in Fig.6(c), where the initial conditions for the solid line correspond to the condition in Eq.(50). A small departure from the condition in Eq.(50) shown by the dotted line in Fig.6(c) prevents the trajectory from approaching $\xi=1$. Thus the internuclear axis $\xi=1$, represents the locus of points of unstable equilibria. In a quantum mechanical treatment, such periodic unstable trajectories is responsible for S-promotion of electron to the the continuum (ionization) when the nuclei approach each other [55]. The potential barrier in Eq.(47) increases when $R$ decreases. As a result, an electron near the top of the barrier slows down and is then collected and promoted to the continuum as the top of the barrier further rises. Due to the strong instability of the locus, a numerical simulation of the corresponding classical trajectory is extremely difficult. [We could not present the classical analog of the 
ionization scenario for S-promotion, in contrast to the T-promotion as shown in Fig. 6(a) and (b).]

The probability of ionization is greatly enhanced in quantum mechanics due to tunnelling into classically forbidden regions of phase space. The cross sections can be calculated using the quasiclassical method, where the probability of transition is given by

$$
P(\rho)=\exp \left(-\frac{2}{\hbar} \operatorname{Im}(S)\right)
$$

where

$$
S(\rho, \epsilon)=\sum_{n} \int_{c} p d R .
$$

Here, $S(\rho, \epsilon)$ is the classical action of the projectile ion, and $p=\sqrt{2 M\left(\epsilon-U(R, \rho)-E_{i}\right)}$ is the projectile momentum, generalized to classically forbidden regions of phase space where $p$ is complex [23]. The integration contour in Eq.(51) is in the complex $R$ plane around the branch points $\left(R_{n}^{c}\right)$ where the initial and final electronic terms cross $\left[E_{f}\left(R_{n}^{c}\right)=E_{i}\left(R_{n}^{c}\right)\right]$. Moreover, $n$ numerates different branch points or channels of ionization for $\mathrm{S}$ and $\mathrm{T}$ promotions. The resulting cross section for hydrogen ionization by collision with a proton is $[53]$

$$
\sigma_{\text {adiabatic }}(v)=\pi v \sum_{n} R_{n}^{2} e^{-2 \Delta_{n} / v}
$$

where $n$ labels many different channels, and the coefficients $\Delta_{n}$ and $R_{n}$ are of order unity in atomic units $\left(R_{n}\right.$ is determined by the branch points $\left.R_{c n}\right)$. In the range of projectile velocities $v=0.4-1$, we find that Eq.(53) can be approximated to within $10 \%$ accuracy by only two exponents with $R_{1}=1.9, \Delta_{1}=0.53$ (corresponding to S-promotion) and $R_{2}=6.7$, $\Delta_{2}=1.8$ (corresponding to T-promotion). Because $\Delta_{1}<<\Delta_{2}$, primarily the S-promotion determines the ionization cross section at small velocities $(v<0.5)$, while both mechanisms contribute to ionization for $v$ in the range $v=0.5-1$.

The new fit predicts an extremely small cross section at very low velocity $\sigma_{\text {fit }}^{\text {low-energy }}(v) \sim$ $\exp \left(-1 / v^{2}\right)$, whereas Eq.(53) gives $\sigma_{\text {adiabatic }}(v) \sim e^{-1.0 / v}$. Therefore, the numerical fit in Eq.(30) underestimates the cross section for $v<0.5$, but gives a result close to the sum in Eq.(53) for $v$ in the range $v=0.5-1$. While the data for hydrogen at very low projectile velocity is absent, and the fit agrees well for the entire dataset in Fig.3, the disagreement is clearly seen when the fit is compared with the experimental data for the ionization of He shown in Fig.6(d). Adiabatic theory results are absent for helium, but the experimental ion- 
ization cross section of He by protons can be described by Eq.(53) with different coefficients $\Delta_{n}$ and $R_{n}$. The behavior of the experimental ionization cross section of He by $\mathrm{He}^{+2}$ is somewhat puzzling because of the very slow decrease of the cross section for small projectile velocity.

In view of these observations, the applicability of the new fit is limited to $v /\left[v_{n l} \sqrt{\left(Z_{p}+1\right)}\right]>0.5$. Note that for small projectile velocity the ionization cross section is ten times smaller than the maximum of the cross section, $\sigma_{\max }$, and the ionization cross section is completely dominated by charge exchange, whose cross section is comparable to $\sigma_{\max }$. Consequently both experimental measurements and theoretical simulations are very difficult for very small projectile velocity.

\section{CONCLUSIONS}

The new scaling in Eq.(26) for the ionization and stripping cross sections of atoms and ions by fully stripped projectiles has been proposed. The new scaling does not have any fitting parameters and describes the shape of the cross section as a single function of the scaled projectile velocity [Eq.(30)]. The proposed scaling formula agrees well with theoretical predictions in the limit of large projectile velocities. The new scaling has been verified by comparison with available experimental data and theoretical simulations for the ionization cross sections of hydrogen and helium by $\mathrm{H}^{+}, \mathrm{He}^{+2}, \mathrm{Li}^{+3}, \mathrm{C}^{+6}$, and $\mathrm{O}^{+8}$. The agreement between the new proposed scaling and experimental data is very good. The difference between the proposed fit and the experimental data is within $15 \%$ accuracy, which is similar to the estimated uncertainty in the measurements. The validity of the fit is limited at very small velocities, where the ionization cross section is very small, about one-tenth of the maximum cross section $\sigma_{\max }$, and the ionization cross section is completely dominated by charge exchange, whose cross section is comparable to $\sigma_{\max }$. Finally, the fit is valid for

scaled projectile velocity $v>0.5 v_{n l} \sqrt{Z_{p}+1}$, where $v_{n l}=v_{0} \sqrt{2 I_{n l} / E_{0}}$ is the orbital velocity of the electron estimated from the ionization potential $I_{n l}$, where $E_{0}=27.2 \mathrm{eV}$ (twice the hydrogen ionization potential). Similarly, the fit is valid for $E>12.5\left(Z_{p}+1\right) I_{n l} / E_{0}$ in units of $k e V / a m u$, where $E$ is the projectile kinetic energy per nucleon.

\section{Acknowledgments}

This research was supported by the U.S. Department of Energy Office of Fusion Energy 
Sciences and the Division of High Energy Physics. It is a pleasure to acknowledge the benefit of useful discussions with Scott Armel-Funkhouser, Larry Grisham, Jun Hasegawa, Ed Lee, Dennis Mueller, David R. Schultz, Ron Olson, Lev Tsendin and Simon Yu.

\section{APPENDIX A: CLASSICAL CROSS SECTION AVERAGED OVER ATOMIC ELECTRON VELOCITY DIRECTIONS}

Gerjuoy averaged the Rutherford cross section over all orientations of the electron velocity $\mathbf{v}_{e}$ (for a fixed electron speed $v_{e}$ ) and derived the differential cross section $d \sigma / d \Delta E\left(v_{e}, v, \Delta E\right.$ ) for energy transfer $\Delta E$ in the collision between a free electron and the projectile [27]. The total cross section is calculated by integrating over values of energy transfer larger than the ionization potential $\left(\Delta E>I_{n l}\right)$ and averaging over the electron velocity distribution function $(\mathrm{EVDF}) f\left(v_{e}\right)$. This gives

$$
\sigma\left(v, I_{n l}, Z_{p}\right)=Z_{p}^{2} \int_{0}^{\infty} \sigma_{I_{n l}}\left(v, v_{e}\right) f\left(v_{e}\right) d v_{e}
$$

where

$$
\sigma_{I_{n l}}\left(v, v_{e}\right)=\int_{I_{n l}}^{\infty} \frac{d \sigma}{d \Delta E}\left(v, v_{e}, \Delta E\right) d \Delta E,
$$

and $d \sigma / d \Delta E\left(v_{e}, v, \Delta E\right)$ is defined by [27]

$$
\frac{d \sigma}{d \Delta E}\left(v, v_{e}, \Delta E\right)=\frac{\pi a_{0}^{2}}{4} \frac{E_{0}^{2}}{\Delta E^{3}} \frac{S\left(v, v_{e}, \Delta E\right)}{v^{2} v_{e}}
$$

where

$$
S\left(v, v_{e}, \Delta E\right)=\left[\begin{array}{c}
\left(v^{2}-v_{e}^{2}\right)\left(v_{e}^{2}-v^{2}-2 \Delta E / m_{e}\right)\left(v_{\text {low }}^{-1}-v_{u p}^{-1}\right)+ \\
2\left(v_{e}^{2}+v^{2}+\Delta E / m_{e}\right)\left(v_{u p}-v_{\text {low }}\right)-1 / 3\left(v_{u p}^{3}-v_{\text {low }}^{3}\right)
\end{array}\right]
$$

Here, $v_{\text {up }}$ and $v_{\text {low }}$ are defined by

$$
\begin{gathered}
v_{u p}=v_{e}+v \\
v_{\text {low }}=\max \left(\left|v_{e}-v\right|, \sqrt{v_{e}^{2}-2 \Delta E / m_{e}}-v\right) .
\end{gathered}
$$

For very large projectile velocities $v>>v_{e}$, it follows that $S \approx 8 v_{e}\left(2 v_{e}^{2} / 3+\Delta E / m_{e}\right)$, and Eq.(A3) yields

$$
\frac{d \sigma_{\text {classical }}^{\text {high energy }}}{d \Delta E}\left(v, v_{e}, \Delta E\right)=2 \pi a_{0}^{2} \frac{E_{0}^{2}}{\Delta E^{3} m_{e} v^{2}}\left(\frac{2 m_{e} v_{e}^{2}}{3}+\Delta E\right) .
$$


Substitution of Eq.(A4) into Eq.(A2), and subsequent substitution of Eq.(A2) and the EVDF Eq.(8) into Eq.(A1) give

$$
\sigma_{\text {classical }}^{\text {high } \text { energy }}\left(v, I_{n l}, Z_{p}\right)=\frac{10}{3} \pi Z_{p}^{2} a_{0}^{2} \frac{v_{0}^{2} E_{0}}{v^{2} I_{n l}}
$$

In the general case with $v \sim v_{e}$, substituting the EVDF Eq.(8) into Eqs.(A2) and (A1) yields

$$
\sigma_{\text {classical }}\left(v, I_{n l}, Z_{p}\right)=\pi a_{0}^{2} E_{0}^{2} \frac{Z_{p}^{2}}{I_{n l}^{2}} G_{\text {classical }}\left(\frac{v}{\sqrt{2 I_{n l} / m_{e}}}\right)
$$

where

$$
G_{\text {classical }}(x)=\frac{1}{x^{2}} \int_{0}^{\infty} \int_{1 / 2}^{\infty} \frac{S\left(x \sqrt{2 I_{n l} / m_{e}}, v_{e}, \Delta E\right) f\left(v_{e}\right)}{\Delta E^{3} v_{e}} d \Delta E d v_{e} .
$$

The approximate formula for $G_{\text {classical }}(x)$ is given below in Eq.(C3).

\section{APPENDIX B: THE BORN APPROXIMATION}

Although the Born approximation is valid only for large projectile velocities $v>>Z_{p} v_{0}$ [23], the Born approximation does give results close to the experimental data even outside its validity range [40]. Therefore, we have studied cross sections in the Born approximation for the entire velocity range.

In the Born approximation, the ionization cross section for hydrogen atoms by impact of fully stripped projectile atoms with charge $Z_{p}$ is given by $[16,31,32]$,

$$
\sigma_{n l}^{B A}(v)=8 \pi a_{0}^{2} Z_{p}^{2} \frac{v_{0}^{2}}{v^{2}} \int_{0}^{\infty} \frac{P_{I_{n l}}(q, v)}{q^{3}} d q,
$$

where $P_{I_{n l}}(q, \widetilde{v})$ is the probability of ionization, and $q m_{e} v_{0}$ is the momentum transfer during the collision. We introducing the velocity in atomic units $\widetilde{v} \equiv v / v_{0}$, and $P_{I_{n l}}(q, \widetilde{v})$ is determined by [31]

$$
P_{I_{n l}}(q, \widetilde{v})=\int_{0}^{\infty} \frac{d P(q, \kappa)}{d \kappa} \Theta\left(q-\frac{\frac{I_{n l}}{E_{0}}+\frac{1}{2} \kappa^{2}}{\widetilde{v}}\right) d \kappa
$$

Here, $\Theta(x)$ is the Heaviside function, and $d P(q, \kappa) / d \kappa$ is the differential probability of ejecting an electron with momentum $\kappa m_{e} v_{0}$ when the momentum transfer from the projectile is $q m_{e} v_{0}$,

$$
\frac{d P(q, \kappa)}{d \kappa}=\left|\left\langle\Psi_{\kappa}^{*}(\mathbf{p}) \Psi_{0}(\mathbf{p}+\mathbf{q})\right\rangle\right|^{2}=\left|\left\langle\Psi_{\kappa}^{*}(\mathbf{r}) e^{i \mathbf{q r}} \Psi_{0}(\mathbf{r})\right\rangle\right|^{2}
$$


In Eq.(B3), $\Psi_{\kappa}^{*}(\mathbf{p})$ and $\Psi_{\kappa}^{*}(\mathbf{r})$ are the wave functions of the continuous spectrum (ionized electron) in momentum space and coordinate space, respectively; $\Psi_{0}(\mathbf{p})$ and $\Psi_{0}(\mathbf{r})$ are the wave functions of the ground state, and star $\left({ }^{*}\right)$ denotes complex conjugate. According to [31],

$$
\frac{d P(q, \kappa)}{d \kappa}=2^{8} \kappa q^{2} \frac{\left[q^{2}+\frac{1}{3}\left(1+\kappa^{2}\right)\right] \exp \left\{-2 / \kappa \arctan \left[2 \kappa /\left(1+q^{2}-\kappa^{2}\right)\right]\right\}}{\left[(q+\kappa)^{2}+1\right]^{3}\left[(q-\kappa)^{2}+1\right]^{3}\left(1-e^{-2 \pi / \kappa}\right)} .
$$

For $q>>1$, the function $d P(q, \kappa) / d \kappa$ has a sharp maximum at $\kappa=q[23]$

$$
\frac{d P(q, \kappa)}{d \kappa}=\frac{8}{3 \pi} \frac{1}{\left[(q-k)^{2}+1\right]^{3}},
$$

which simply means that the entire momentum $q$ is transferred to the ionized electron momentum $\kappa$. At small $q<1, d P(q, \kappa) / d \kappa \sim \kappa q^{2}$ and the width of the function $P(q, \kappa)$ as a function of $\kappa$ is of order unity in atomic units.

For large projectile velocity $v>>v_{0}$, considerable simplification can be made by neglecting the electron kinetic energy $\frac{1}{2} \kappa^{2}$ in the argument of the Heaviside function in Eq.(B2). The approximation

$$
\Theta\left(q-\frac{\frac{I_{n l}}{E_{0}}+\frac{1}{2} \kappa^{2}}{\frac{v}{v_{0}}}\right) \rightarrow \Theta\left(q-\frac{\frac{I_{n l}}{E_{0}}}{\frac{v}{v_{0}}}\right)
$$

is referred to as the close-coupling approximation. In this case, $P(q, v)$ can be characterized by a function of one argument, $S_{i n h}(q)$, with

$$
P_{I_{n l}}(q, \widetilde{v})=S_{i n h}(q) \Theta\left(q-\frac{v_{0} I_{n l}}{v E_{0}}\right),
$$

where

$$
S_{i n h}(q)=\int_{0}^{\infty} \frac{d P(q, \kappa)}{d \kappa} d \kappa .
$$

The function $S_{i n h}(q)$ is refereed to as the total ionization transition strength [39]. Substituting Eq.(B6) results in artificial, additional contributions to the integral in Eq.(B2) for $\kappa>\kappa_{\text {add }}=\sqrt{2\left(q v / v_{0}-I_{n l} / E_{0}\right)}$. For large projectile velocities $v>>v_{0}$ and $q>>1$, $\kappa_{\text {add }} \simeq \sqrt{2 q v / v_{0}}$. The function $d P(q, \kappa) / d \kappa$ has a sharp maximum at $\kappa=q$ [see Eq.(B5)]. Therefore the artificial additions for $\kappa>\kappa_{\text {add }}$ do not contribute to the integral if $\kappa_{\text {add }}>q$, which corresponds to $q<2 v$, and the substitution in Eq.(B6) is valid. In the opposite case of large projectile velocities $v>>v_{0}$ but small $q$, it follows that $q \sim v_{0} I_{n l} /\left(v E_{0}\right)<<1$, for the range of $q \kappa_{\text {add }} \sim 1$, and the function $d P(q, \kappa) / d \kappa$ decreases rapidly for $\kappa>1$. Therefore, the artificial additions for $\kappa>\kappa_{\text {add }}$ do not contribute to the integral if $\kappa_{\text {add }}>1$. Hence, the substitution in Eq.(B6) is valid for $v>>v_{0}$. Figure 7 shows plots of $P_{I_{n l}}(q, \widetilde{v})$ [Eq.(B2)] and 


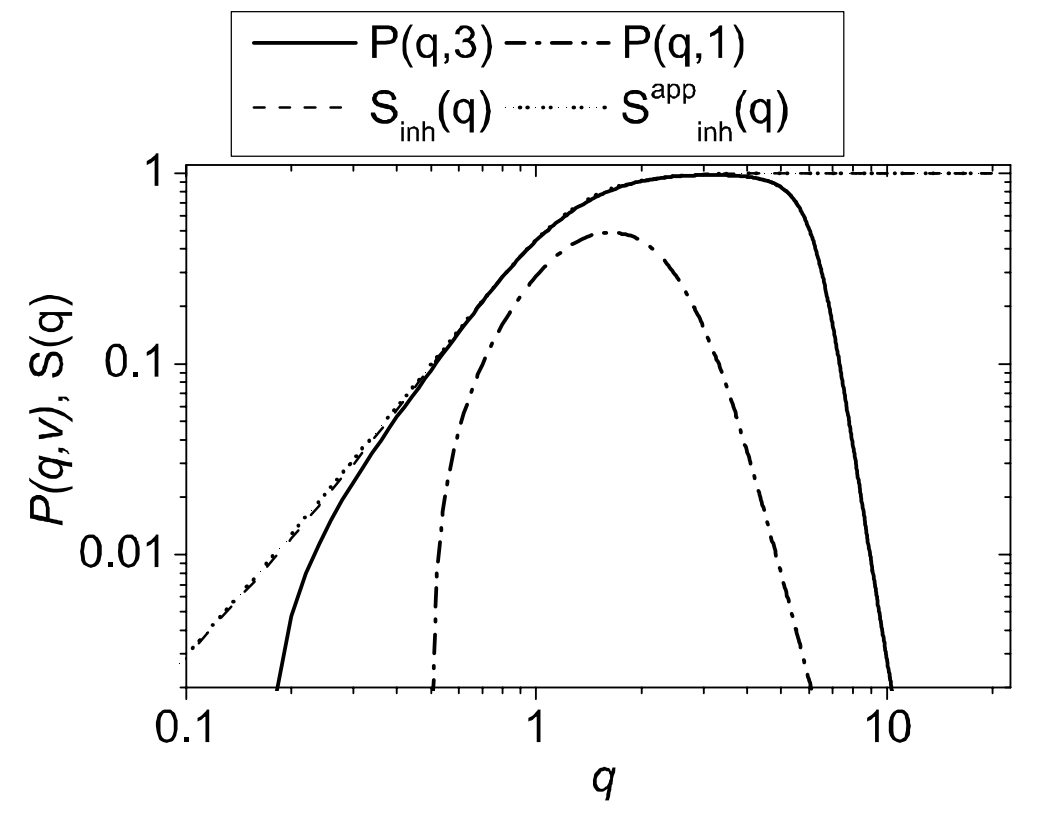

FIG. 7: Total ionization transition strength for atomic hydrogen as a function of transferred momentum $q$. The exact function $P(q, v)$ [Eq.( B2)] for $\widetilde{v}=1$ and $\widetilde{v}=3$ is compared with the approximate function $S_{i n h}(q)$ [Eq.(B8)] (which is independent of $v$ ) and the fit $S_{i n h}^{a p p}(q)$ in Eq.(B9).

$S_{i n h}(q)\left[\right.$ Eq.(B8)] for $\widetilde{v}=1$ and $\widetilde{v}=3$. At small projectile velocities $v<v_{0}$, the substitution in Eq.(B6) produces a considerable error [see Fig.7]. For repetitive calculations, the function $S_{i n h}(q)$ in Eq.(B8) can be approximated to within $3 \%$ accuracy by

$$
S_{\text {inh }}^{a p p}(q)=\left[\begin{array}{cc}
\frac{0.545 q^{2}}{(q-0.9)^{2}+1.21} & q<2 \\
\tanh (0.8 q) & q \geq 2
\end{array}\right]
$$

The functions $S_{i n h}(q)$ [Eq.(B8)] and $S_{i n h}^{a p p}(q)$ [Eq.(B9)] are shown in Fig. 7.

Having estimated the function $P_{I_{n l}}(q, \widetilde{v})$, the total cross section can be evaluated analytically for large $v>>v_{0}$. The region of small $q$ contributes significantly to the cross section [see Eq.(B1)]. Therefore, we split the integration in Eq.(B1) into the two regions $q<q_{u p}$ and $q>q_{u p}$, where $q_{u p}=1 / 2$. In the first region $q<q_{u p}$, it follows that $P_{I_{n l}}(q, v) \approx S_{i n h}^{a p p}(q) \approx 0.283 q^{2}$, and the integration in Eq.(B1) gives

$$
\int_{0}^{q_{u p}} d q \frac{P_{I_{n l}}(q, v)}{q^{3}} \approx \int_{q_{\min }}^{q_{u p}} d q \frac{0.283}{q}=0.283 \ln \left(q_{u p} / q_{\min }\right),
$$

where $q_{\min }=v_{0} I_{n l} / v E_{0}$. In the second region, only the range of $q_{u p}<q<2$ contributes to the integral, because at large $q>>1, P_{I_{n l}}(q, v) / q^{3} \approx 1 / q^{3}$ and the contribution to 
the integral for large $q$ quickly decreases to zero. At very large $q>2 v, P_{I_{n l}}(q, v)$ became smaller than unity, but this region does not contribute to the integral and can be neglected. As a result, the integral $\int_{q_{u p}}^{\infty} d q P_{I_{n l}}(q, v) / q^{3}$ does not depend on $v$ (for the large $v$ under consideration). The integration from $q_{u p}$ to infinity gives $\int_{q_{u p}}^{\infty} d q P_{I_{n l}}(q, v) / q^{3} \approx 0.666$, and finally the result is similar to the Bethe formula in Eq.(11) with

$$
\sigma^{\text {Bethe }}(\widetilde{v})=8 \pi a_{0}^{2} \frac{Z_{p}^{2}}{\widetilde{v}^{2}}[0.283 \ln (\widetilde{v})+0.666] .
$$

The small differences from the Bethe formula are due to utilization of the close coupled approximation in Eq.(B10), which overestimates $P_{I_{n l}}(q, v)$ at small $q$ [see Fig.7].

Comparison with the exact calculation (Fig.1) shows that the Bethe asymptotic result is close to the exact calculation in Eq.(B1) for $\widetilde{v}>2$. To extend the Bethe formula to lower velocities, the second-order correction in the parameter $v_{0} / v$ has been calculated in [37], yielding the cross section in the form

$$
\sigma_{\text {mod }}^{\text {Bethe }}(\widetilde{v})=4 \pi a_{0}^{2} \frac{Z_{p}^{2}}{\widetilde{v}^{2}}\left[0.57 \ln (\widetilde{v})+1.26-0.66 \frac{1}{\widetilde{v}^{2}}\right],
$$

where $\widetilde{v}=v / v_{0}$. Equation (B12) agrees with the exact calculation in Eq.(B1) to within $10 \%$ for $\widetilde{v}>1.1$. We have developed the following fit for the cross section in the Born approximation,

$$
\sigma_{\text {fit }}^{B A}(\widetilde{v})=4 \pi a_{0}^{2} \frac{Z_{p}^{2}}{\widetilde{v}^{2}}\left[0.283 \ln \left(\widetilde{v}^{2}+1\right)+1.26\right] \exp \left[-\frac{1.95}{\widetilde{v}\left(1+1.2 \widetilde{v}^{2}\right)}\right],
$$

which agrees with the exact calculation in Eq.(B1) to within $2 \%$ for $\widetilde{v}>1$, and to within $20 \%$ for $0.2<\widetilde{v}<1$.

The previous analysis was performed for the hydrogen atom. In the case of hydrogen-like electron orbitals, the similarity principle can be used. The quantity $d P(q, \kappa) / d \kappa$ is identical for different electron orbitals if $q, \kappa$ are scaled with the factor $1 / Z_{T}=v_{0} / v_{n l}$ [23]. Therefore, $P_{n l}(q, v)=P_{H}\left(q v_{0} / v_{n l}, v / v_{n l}\right)$, where $H$ denotes hydrogen atom, and

$$
\sigma_{f i t}^{B A}\left(\widetilde{v}=\frac{v}{v_{n l}}\right)=4 \pi a_{0}^{2} \frac{v_{0}^{4}}{v_{n l}^{4}} \frac{Z_{p}^{2}}{\widetilde{v}^{2}}\left[0.283 \ln \left(\widetilde{v}^{2}+1\right)+1.26\right] \exp \left[-\frac{1.95}{\widetilde{v}\left(1+1.2 \widetilde{v}^{2}\right)}\right],
$$

where

$$
\widetilde{v}=\frac{v}{v_{n l}}=\frac{v}{\sqrt{2 I_{n l} / m_{e}}}
$$

As we have noted for helium, most scalings can be used even for non-hydrogen-like electron orbitals, provided the relationship in Eq.(B15) is used. 

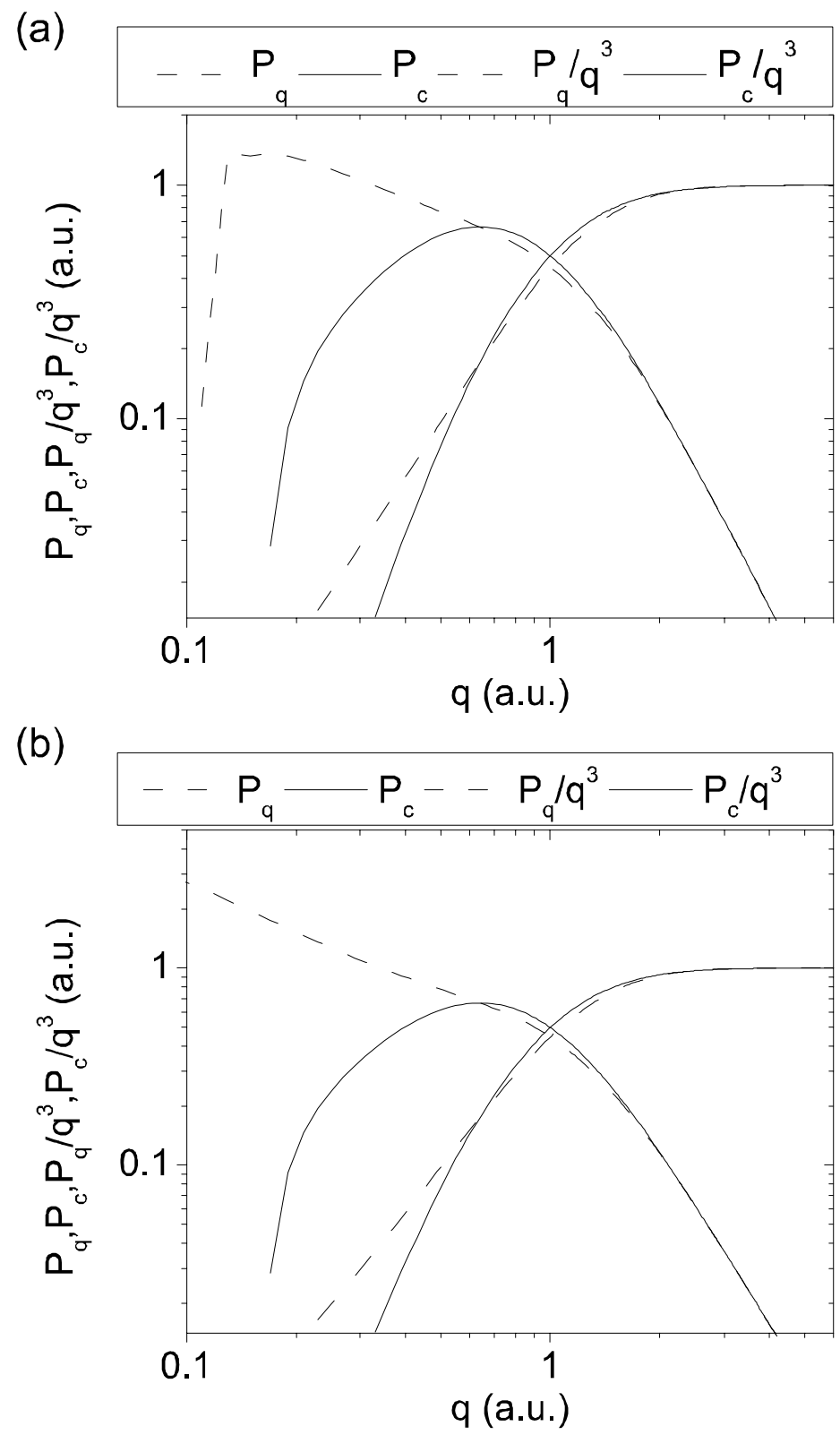

FIG. 8: Probability of ionization of atomic hydrogen as a function of transferred momentum; $P_{c}(q)$ is given by classical mechanics [Eq.( B2)], and $P_{q}(q, v)$ is given by quantum mechanics [Eq.(B30)]. The plots correspond to (a) $\widetilde{v}=5$ and (b) $\widetilde{v}=15$.

\section{Comparison between the quantum mechanical and classical trajectory calcula-} tions for $v>>v_{n l}$

We have previously noted that the classical trajectory calculation underestimates the ionization cross section at large velocities $v>>v_{n l}$. To compare the ionization cross section 
calculated in the classical trajectory and Born approximations, we present both cross sections in the form of Eq.(B1). In the limit $v>>v_{n l}$, the momentum transferred to the electron during a collision with impact parameter $\rho$ is given by Eq.(1), i.e.,

$$
q_{x}(\rho) \equiv m_{e} \Delta v_{x}(\rho)=\frac{2 e^{2} Z_{p}}{v \rho}
$$

where $x$-axis is chosen in the direction perpendicular to the projectile ion trajectory along the momentum transfer. Because $v>>v_{n l}$, the electron velocity is neglected in Eq.(B16). In classical mechanics, ionization occurs if the energy transfer to the electron is more than the ionization potential, $\left[\left(m_{e} \mathbf{v}_{e}+\mathbf{q}\right)^{2}-m_{e}^{2} v^{2}\right] / 2 m_{e}>I_{n l}$.

A small momentum transfer to the electron along the projectile trajectory $q_{z}(\rho)$ can be determined making use of the energy conservation. Due to conservation of the momentum, the momentum transferred from the projectile particle is $-q_{z}(\rho)$. The projectile energy change is $\left[(M \mathbf{v}-\mathbf{q})^{2}-M^{2} v^{2}\right] / 2 M=-v q_{z}$. Conservation of energy gives

$$
v q_{z} \equiv \frac{1}{2 m_{e}}\left[\left(m_{e} \mathbf{v}_{e}+\mathbf{q}\right)^{2}-m_{e}^{2} v_{e}^{2}\right]
$$

In the limit $v>>v_{e}$, it follows that $q_{z}<<q_{x}$, and consequently the total transferred momentum to the electron is $q=\sqrt{q_{\mathbf{x}}^{2}+q_{z}^{2}} \simeq q_{x}$. The momentum of the ejected electron can be determined from the energy conservation relation

$$
\kappa^{2} / 2 m_{e}=\left[\left(m_{e} \mathbf{v}_{e}+\mathbf{q}\right)^{2}-m_{e}^{2} v_{e}^{2}\right] / 2 m_{e}-I_{n l}
$$

In classical mechanics, the ionization probability of the ejected electron with momentum $\kappa$ in a collision with total momentum transfer $q$ is given by the integral over the electron distribution function,

$$
\frac{d P_{c}(q, \kappa)}{d \kappa}=\frac{\kappa}{m_{e}} \int f\left(\mathbf{v}_{e}\right) \mathbf{d} \mathbf{v}_{e} \delta\left(\frac{\kappa^{2}}{2 m_{e}}-q_{x} v_{x}-\frac{q^{2}}{2 m_{e}}-I_{n l}\right) .
$$

Introducing the one-dimensional electron distribution function

$$
f_{x}\left(v_{e x}\right)=\int f\left(\mathbf{v}_{e}\right) d v_{y} d v_{z}
$$

and substituting $q \simeq q_{x}$, Eq.(B19) simplifies to become

$$
\frac{d P_{c}(q, \kappa)}{d \kappa}=\frac{\kappa}{q m_{e}} f_{x}\left(\frac{\kappa^{2}-q^{2}-2 m_{e} I_{n l}}{2 q m_{e}}\right) .
$$


For hydrogen-like electron orbitals given by Eq. $(8), f_{x}\left(v_{e x}\right)$ can be readily calculated to be

$$
f_{x}\left(v_{e x}\right)=\frac{8}{3 \pi} \frac{v_{n l}^{5}}{\left[v_{e x}^{2}+v_{n l}^{2}\right]^{3}} .
$$

Substituting the hydrogen-like electron distribution function Eq.(B22) into Eq.(B29) gives in atomic units

$$
\frac{d P_{c}(q, \kappa)}{d \kappa}=\frac{16 \kappa}{3 \pi} \frac{\left(2 q m_{e}\right)^{5} v_{n l}^{5}}{\left[\left(\kappa^{2}-q^{2}-2 m_{e} I_{n l}\right)^{2}+\left(2 q m_{e} v_{n l}\right)^{2}\right]^{3}} .
$$

Let us compare Eq.(B23) with the quantum mechanical result Eq.(B5). In the limit $q>>1$, $\kappa \approx q$ and the two functions are equivalent. Both functions $d P(q, \kappa) / d \kappa$ have a maximum at $\kappa=q$, and the width of the maximum is of order 1 , which simply means that the entire momentum $q$ is transferred to the ionized electron momentum $\kappa$.

Moreover it is possible to prove that the classical mechanical $d P_{c}(q, \kappa) / d \kappa$ is equivalent to the quantum mechanical function $d P_{q}(q, \kappa) / d \kappa$ for any $s$-electron orbital (spherically symmetrical wave function). Indeed, for large $k>>1$, the ejected electron can be described as a sum over plane waves $\Psi_{\kappa}^{*}(\mathbf{r}) \approx e^{i \mathbf{k r}}$, and substituting $\Psi_{\kappa}^{*}(\mathbf{r})$ into Eq.(B3) gives

$$
\frac{d P_{q}(q, \kappa)}{d \kappa}=\frac{1}{(2 \pi \hbar)^{3}} \int\left|\left\langle e^{i(\mathbf{q}-\mathbf{k}) \mathbf{r} / \hbar} \Psi_{0}(\mathbf{r})\right\rangle\right|^{2} k^{2} d o_{\mathbf{k}}=\frac{1}{m_{e}^{3}} \int f\left(\frac{\mathbf{q}-\mathbf{k}}{m_{e}}\right) k^{2} d o_{\mathbf{k}},
$$

where integral over $d o_{\mathbf{k}}=2 \pi \sin \vartheta d \vartheta$ designates averaging over all directions of the $\mathbf{k}$-vector, $\vartheta$ is the angle between $\mathbf{q}$ and $\mathbf{k}$, and $f\left(v_{e}\right)$ is the electron distribution function in velocity space. Note that $|\mathbf{q}-\mathbf{k}|^{2}=\mathbf{q}^{2}+\mathbf{k}^{2}-2 \mathbf{q} \cdot \mathbf{k}=(q-k)^{2}+4 q k \sin \vartheta / 2^{2}$. In the limit $q>>1$, $k \approx q$ and only small $\vartheta$ contribute to the integral in Eq.(B24). Therefore, averaging over all directions of the $\mathbf{k}$-vector gives

$$
\frac{1}{m_{e}^{2}} \int f\left(\frac{\mathbf{q}-\mathbf{k}}{m_{e}}\right) k^{2} d o_{\mathbf{k}}=\frac{1}{m_{e}^{2}} \int f\left(\frac{\sqrt{(q-k)^{2}+q k \vartheta^{2}}}{m_{e}}\right) 2 \pi k^{2} \vartheta d \vartheta .
$$

Introducing $v_{\perp}=k \vartheta / m_{e}$, the integral in Eq.(B25) takes form

$$
\int f\left(\sqrt{\left(\frac{q-k}{m_{e}}\right)^{2}+v_{\perp}^{2}}\right) d^{2} v_{\perp}=f_{x}\left(\frac{q-k}{m_{e}}\right),
$$

where $f_{x}$ is the one-dimensional electron velocity distribution function. Substituting Eqs.(B26) and (B25) into Eq.(B24) yields

$$
\frac{d P_{q}(q, \kappa)}{d \kappa}=\frac{1}{m_{e}} f_{x}\left(\frac{q-k}{m_{e}}\right) .
$$


Note that in the limit $q>>m_{e} v_{n l}$, it follows that $\kappa \approx q$, and Eq.(B21) becomes

$$
\frac{d P_{c}(q, \kappa)}{d \kappa}=\frac{1}{m_{e}} f_{x}\left(\frac{q-k}{m_{e}}\right) .
$$

Finally, comparing Eqs.(B27) and (B28) we arrive at the equivalence of functions $d P(q, \kappa) / d \kappa$ in quantum mechanics and classical mechanics in the limit $q>>m_{e} v_{n l}$.

The situation is completely different for small $q<<m_{e} v_{n l}$. From Eq.(B23) it follows that $d P_{c}(q, \kappa) / d \kappa \sim \kappa q^{5}$, and $d P_{c}(q, \kappa) / d \kappa$ is much smaller than $d P_{q}(q, \kappa) / d \kappa \sim \kappa q^{2}$. Therefore, classical mechanics strongly underestimates the probability of ionization for small transferred momentum $q<m_{e} v_{n l}$.

The total probability of ionization in classical mechanics is

$$
P_{c}(q)=\int_{0}^{\infty} d \kappa \frac{d P_{q}(q, \kappa)}{d \kappa}=\int \Theta\left(q v_{e x}+\frac{q^{2}}{2 m_{e}}-I_{n l}\right) f\left(\mathbf{v}_{e}\right) \mathbf{d} \mathbf{v}_{e}
$$

Equation (B29) simplifies to become

$$
P_{c}(q)=\int \Theta\left(q v_{e x}+\frac{q^{2}}{2 m_{e}}-I_{n l}\right) f_{x}\left(v_{e x}\right) d v_{e x}
$$

The differential cross section for momentum transfer $q$ is given by

$$
d \sigma_{c}(q)=2 \pi \rho(q) d \rho(q)
$$

where $\rho(q)$ is given by Eq.(B16). Substituting $\rho(q)$ from Eq.(B16) into Eq.(B31) gives

$$
d \sigma_{c}(q)=\frac{8 \pi e^{4} Z_{p}^{2}}{v^{2} q^{3}} d q
$$

which is the Rutherford differential cross section for scattering at small angles. Finally, the total ionization cross section is

$$
\sigma_{c}=8 \pi a_{0}^{2} Z_{p}^{2} \frac{v_{0}^{2}}{v^{2}} \int_{I_{n l} / v}^{\infty} \frac{P_{c}(q)}{q^{3}} d q .
$$

In Eq. (B33), we accounted for the fact that the minimum $q$ is $q=I_{n l} / v$. Note that in the region $q=[1-3] I_{n l} / v$ ionization occurs due the collisions with very fast electrons $v_{e} \sim v$, and $q_{x} \sim q_{z}$. The previous analysis which assumed $v_{e}<<v$ and $q_{x}>>q_{z}$ is not valid in this region of extremely small $q$. However, because $P_{c}(q) / q^{3} \rightarrow 0$ as $q \rightarrow 0$, this region of $q=$ $[1-3] I_{n l} / v$ does not contribute to the integral in Eq. (B33) and can be neglected. Moreover such small momentum transfers correspond to very large impact parameter $\rho / v \sim a_{n l} / v_{n l}$, 
where the collision becomes adiabatic. Therefore, accurate calculations yield even smaller $P_{c}(q)$ than in Eq.(B30).

Equation (B33) is identical to Eq.(B1), where the quantum mechanical ionization probability $P_{q}(q, v)$ is replaced by the classical mechanical ionization probability $P_{c}(q)$ in Eq.(B30). The functions $P_{q}(q, v)$ [Eq.(B2)] and $P_{c}(q)$ [Eq.(B30)] are shown in Fig.8. Figure 8 shows that the functions $P_{I_{n l}}(q, v)$ and $P_{c}(q)$ are nearly identical for $q>0.6$. The classical probability of ionization $P_{c}(q)$ rapidly tends to zero for $q<0.6$, while the quantum probability of ionization, $P_{q}(q) \approx 0.283 q^{2}$, is much larger than $P_{c}(q)$ at small $q$. The cross section is

determined by $P_{q}(q) / q^{3}$. Therefore the region of small $q$ contributes considerably to the quantum mechanical cross section. Note that $P_{q}(q) / q^{3} \rightarrow 0$ as $q \rightarrow I_{n l} / \widetilde{v}$, and for larger $\widetilde{v}$, $I_{n l} / \widetilde{v}$ is smaller. It follows that the region of small $q$ contributes more to the cross section [compare Fig.8(a) for $\widetilde{v}=5$, and Fig.8(b) for $\widetilde{v}=15$ ]. For $\widetilde{v}=5$, the classical mechanical ionization cross section in atomic units is $\sigma_{c}=0.23$, and the quantum mechanical ionization cross section is $\sigma_{q}=0.30$, which is $30 \%$ larger than the classical mechanical cross section. For $\widetilde{v}=15, \sigma_{c}=0.025$ and $\sigma_{q}=0.043$, which is $70 \%$ larger.

\section{APPENDIX C: FORMULARY FOR IONIZATION CROSS SECTION}

In the high energy limit of fast projectile motion $v>>v_{n l}$, the classical mechanical calculation can be readily carried out (see Appendix A).

The Bohr formula [26] neglects the electron velocity in the atom completely, which gives

$$
\sigma^{B o h r}\left(v, I_{n l}, Z_{p}\right)=2 \pi Z_{p}^{2} a_{0}^{2} \frac{v_{0}^{2} E_{0}}{v^{2} I_{n l}} .
$$

Accounting for the electron velocity gives an additional factor of $5 / 3$ compared with the Bohr formula. This gives the classical mechanical ionization cross section in the limit of high projectile velocity

$$
\sigma_{\text {classical }}^{\text {high energy }}\left(v, I_{n l}, Z_{p}\right)=\frac{5}{3} 2 \pi Z_{p}^{2} a_{0}^{2} \frac{v_{0}^{2} E_{0}}{v^{2} I_{n l}}
$$

In the general case with $v \sim v_{n l}$, the classical mechanical calculation accounting for the finite electron velocity in the atom, but neglecting the influence of the target nucleus on the 
electron has been performed by Gerjuoy [27] [see Appendix A]. This gives

$$
\sigma^{G G V}\left(v, I_{n l}, Z_{p}\right)=\pi a_{0}^{2} E_{0}^{2} \frac{Z_{p}^{2}}{I_{n l}^{2}} G^{G G V}\left(\frac{v}{\sqrt{2 I_{n l} / m_{e}}}\right)
$$

The tabulation of the function $G^{G G V}(x)$ is presented in Ref.[29] for $x>1$, and in Ref.[30] for $x<1$, which gives

$$
G^{G G V}(x)=\left\{\begin{array}{c}
\frac{g(x)}{4 x^{2}} \quad \text { for } \quad x>1 \\
\frac{0.696}{\exp \left(\frac{0.585-x}{0.096}\right)+1} \quad \text { for } \quad x<1
\end{array}\right\}
$$

where

$$
g(x)=\left\{\begin{array}{c}
\frac{35}{6}+\frac{35}{3 \pi} \arctan c+\frac{128\left(x^{3} b^{3}-b^{3 / 2}\right)}{9 \pi}+\frac{b c}{3 \pi}\left(35-\frac{58 b}{3}-\frac{8 b^{2}}{3}\right)+ \\
\frac{2 a b x}{3 \pi}\left[\left(5-4 x^{2}\right)\left(3 a^{2}+1.5 a b+b^{2}\right)-c x(7.5+9 a+5 b)\right]- \\
\frac{16}{\pi} x a^{4} \ln \left(4 x^{2}+1\right)-a x^{2}\left(1+\frac{2 \arctan c}{\pi}\right)\left(2.5+3 a+4 a^{2}+8 a^{3}\right)
\end{array}\right\}
$$

and

$$
a=1 /\left(1+x^{2}\right) \quad c=3 x / 4 \quad b=1 /\left(1+c^{2}\right) .
$$

Gryzinski's approximation for the ionization cross section [20] expressed in the form of Eq.(C2) is given by

$$
\sigma^{G r y z}\left(v, I_{n l}, Z_{p}\right)=\pi a_{0}^{2} E_{0}^{2} \frac{Z_{p}^{2}}{I_{n l}^{2}} G^{G r y z}\left(\frac{v}{\sqrt{2 I_{n l} / m_{e}}}\right)
$$

where

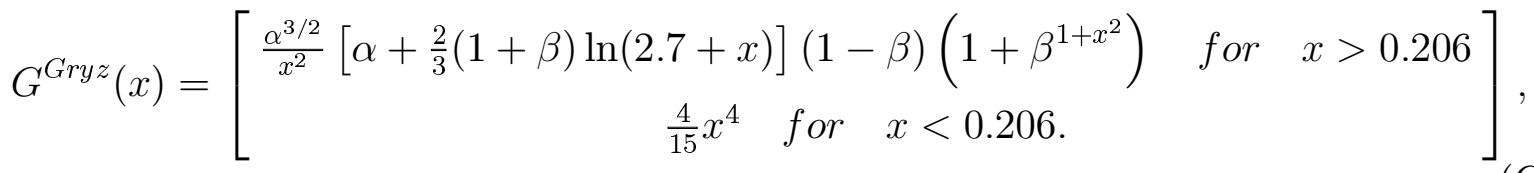

and $\alpha=x^{2} /\left(1+x^{2}\right) \beta=1 /[4 x(1+x)]$.

Bethe's asymptotic quantum mechanical calculation in the Born approximation [31] is valid for $v / v_{0}>2 Z_{p}$ and $v>>v_{n l}$ [23], and can be expressed as

$$
\sigma^{\text {Bethe }}=4 \pi a_{0}^{2} \quad \frac{v_{0}^{4} Z_{p}^{2}}{v^{2} v_{n l}^{2}} \cdot\left[0.57 \ln \left(\frac{v}{v_{n l}}\right)+1.26\right] .
$$

The region of validity of the Born approximation and, hence, the Bethe formula is [23, 24]

$$
v>\max \left(2 Z_{p} v_{0}, v_{n l}\right)
$$


The first condition in Eq. (C8a) assures that the projectile potential is taken into account in the Born approximation; the second condition allows use of the unperturbed atomic wave function.

To describe the behavior of the cross section near the maximum, the second-order correction in the parameter $v_{n l} / v$ has been calculated in Ref.[37], yielding the cross section in the form

$$
\sigma_{\text {mod }}^{\text {Bethe }}(\widetilde{v})=4 \pi a_{n l}^{2} \frac{v_{0}^{2}}{v_{n l}^{2}} \frac{Z_{p}^{2}}{\widetilde{v}^{2}}\left[0.566 \ln (\widetilde{v})+1.26-0.66 \frac{1}{\widetilde{v}^{2}}\right]
$$

where

$$
\widetilde{v}=\frac{v}{v_{n l}}=\frac{v}{\sqrt{2 I_{n l} / m_{e}}}, a_{n l}^{2}=a_{0}^{2} \frac{E_{0}}{2 I_{n l}} .
$$

In the general case with $v \sim v_{n l}$, the ionization cross section in the Born approximation was first calculated in Ref.[40]. We have developed the following fit for the Bates and Griffing result

$$
\sigma_{f i t}^{B A}\left(\widetilde{v}=\frac{v}{v_{n l}}\right)=4 \pi a_{n l}^{2} \frac{v_{0}^{2}}{v_{n l}^{2}} \frac{Z_{p}^{2}}{\widetilde{v}^{2}}\left[0.283 \ln \left(\widetilde{v}^{2}+1\right)+1.26\right] \exp \left[-\frac{1.95}{\widetilde{v}\left(1+1.2 \widetilde{v}^{2}\right)}\right] .
$$

The Bethe cross section valid for relativistic particles [32] is given by

$$
\sigma_{\text {rel }}^{\text {Bethe }}=4 \pi a_{n l}^{2} \frac{v_{0}^{2}}{v_{n l}^{2}} \frac{v_{n l}^{2} Z_{p}^{2}}{v^{2}}\left\{M_{i o n}^{2}\left[2 \ln \left(\gamma_{p} \beta_{p}\right)-\beta^{2}\right]+C_{i o n}\right\}
$$

where $\beta_{p}^{2}=v_{p} / c, c$ is the speed of light, $\gamma_{p}=1 / \sqrt{1-\beta_{p}^{2}}$, and $M_{i o n}^{2}$ and $C_{i o n}$ are characteristic constants depending on the ionized atom or ion. For the hydrogen atom, $M_{i o n}^{2}=0.283$ and $C_{\text {ion }}=4.04$.

Gillespie's fit for the ionization cross sections [39] is given by

$$
\sigma^{\text {Gill }}=\exp \left[-\lambda_{n l}\left(v_{0} \sqrt{Z_{p}} / v\right)^{2}\right] \sigma_{\text {mod }}^{\text {Bethe }},
$$

where $\lambda_{n l}$ is a characteristic constant of the ionized atom or ion (for example, for the ground state of atomic hydrogen, $\left.\lambda_{n l}=0.76\right)$, and $\sigma_{\text {mod }}^{\text {Bethe }}$ is the modified Bethe cross section defined in Eq.(C9).

The Olson scaling [42] for the total electron loss cross section $\sigma^{e l}$, which includes both the charge exchange cross section $\sigma^{c e}$ and the ionization cross section, is given by

$$
\sigma^{e l}\left(v, Z_{p}\right)=\pi a_{0}^{2} Z_{p} A_{n l} f^{\text {Olson }}\left(\frac{v}{v_{0} \gamma_{n l} \sqrt{Z_{p}}}\right)
$$


where $f(x)$ describes the scaled cross sections

$$
f^{\text {Olson }}(x)=\frac{1}{x^{2}}\left[1-\exp \left(-x^{2}\right)\right]
$$

and $\gamma_{n l}$ and $A_{n l}$ are constants. For example, $\gamma_{H}=\sqrt{5 / 4}=1.12$ and $A_{H}=16 / 3$ for atomic hydrogen, whereas $\gamma_{H e}=1.44$ and $A_{h e}=3.57$ for helium.

Rost and Pattard [45] proposed a fit for the ionization cross section, which utilizes two fitting parameters, namely the maximum value of the cross section and projectile energy corresponding to the maximum value of the cross section. They showed that if both the cross section and the projectile velocity are normalized to the values of the cross section and the projectile velocity at the cross section maximum, then the scaled cross section $\sigma / \sigma_{\max }$ is well described by the fitting function [45]

$$
\sigma(v)=\sigma_{\max } \frac{\exp \left(-v_{\max }^{2} / v^{2}+1\right)}{v^{2} / v_{\max }^{2}},
$$

where $\sigma_{\max }$ is the maximum cross section, which occurs at the velocity $v_{\max }$.

We have shown that for ionization by a bare projectile, the values $\sigma_{\max }$ and $v_{\max }$ are well defined by the projectile charge $Z_{p}$, with

$$
\begin{aligned}
\sigma_{\max } & =\pi a_{0}^{2} B_{n l} \frac{Z_{p}^{2}}{\left(Z_{p}+1\right)} \frac{E_{0}^{2}}{I_{n l}^{2}}, \\
v_{\max } & =v_{n l} \sqrt{Z_{p}+1}
\end{aligned}
$$

where the coefficient $B_{n l}$ depends weakly on the projectile charge. For example, for ionization of hydrogen by protons, $B_{n l}=0.8$, and for ionization of hydrogen by bare nuclei of helium or lithium, $B_{n l}=0.93$.

Equation (C14) describes well the cross sections at small and intermediate energies, but underestimates the cross section at high energies, because it does not reproduce the logarithmic term of the Bethe formula in Eq. (C7). To improve the agreement with the experimental data and the Bethe formula, we propose the new scaling

$$
\sigma^{i o n}\left(v, I_{n l}, Z_{p}\right)=\pi a_{0}^{2} \frac{Z_{p}^{2}}{\left(Z_{p}+1\right)} \frac{E_{0}^{2}}{I_{n l}^{2}} G^{\text {new }}\left(\frac{v}{v_{n l} \sqrt{Z_{p}+1}}\right)
$$

where

$$
G^{\text {new }}(x)=\frac{\exp \left(-1 / x^{2}\right)}{x^{2}}\left[1.26+0.283 \ln \left(2 x^{2}+25\right)\right]
$$


In all previous equations cross section are given per electron in the orbital. If $N_{n l}$ is the number of electrons in the orbital, the ionization cross section of any electron in the orbital should be increased by the factor $N_{n l}$.

[1] B.G. Logan, C.M. Celata, J.W. Kwan, E.P. Lee, M. Leitner, P.A. Seidl, S.S. Yu, J.J. Barnard, A. Friedman, W.R. Meier, and R.C. Davidson, Laser and Particle Beams 20, 369 (2002).

[2] G.M. Keating and S.W. Bougher, J. Geophys. Res.- Space Phys. 97 (A4), 4189 (1992).

[3] H. Beyer, V.P. Shevelko (eds), Atomic physics with Heavy Ions (Springer, Berlin 1999).

[4] A. Bogaerts, R. Gijbels, and R.J. Carman, Spectrochimica Acta Part B - Atomic Spectroscopy 53, 1679 (1998).

[5] C. Stockl, O. Boine-Frankenheim, M. Geissel, M. Roth, H. Wetzler, W. Seelig, O. Iwase, P. Spiller, R. Bock, W. Suss, and D.H.H. Hoffmann, Nucl. Instrum. Meth.A 415, 558 (1998).

[6] S. Datz, G.W. F. Drake, T.F. galagher, H. Kleinpoppen, and G. Zu Putlitz, Rev. Mod. Phys. 71, S223, (1999).

[7] P. Chen, Part. Accel. 20, 171 (1987); P. Chen, J.J. Su, T. Katsouleas, S. Qilks, and J. M. Dawson, IEEE Trans. on Plasma Science PS.-15, 218 (1987).

[8] R. Govil, W.P. Leemans, E. Yu. Backhaus and J.S. Wurtele, Phys. Rev. Lett. 83, 3202 (1999).

[9] S. Rajagopalan, D.B. Cline, and P. Chen, Nucl. Instrum. Meth.A 355, 169 (1995).

[10] T. Tauschwitz, S.S. Yu, S. Eylon, L. Reginato, W. Leemans, J.O. Rasmussen, and R.O. Bangerter J. Fusion Engineering and Design 32-33, 493 (1996).

[11] M. Roth, T.E. Cowan, M.H. Key, S.P. Hatchett, et al., Phys. Rev. Lett. 86, 436 (2001); M. Tabak, J. Hammer, M. E. Glinsky, W. L. Kruer, S. C. Wilks, J. Woodworth, E. M. Campbell, M. D. Perry, and R. J. Mason, Phys. Plasmas 1, 1626 (1994).

[12] G. S. Voronov, Atomic Data and Nuclear Data Tables, 65, 1, (1997).

[13] M.E. Rudd, Y.-K. Kim, D.H. Madison, and J.W. Galallagher, Rev. Mod. Phys. 64, 441 (1992).

[14] M.E. Rudd, Y.-K. Kim, D.H. Madison, and T.J. Gay, Rev. Mod. Phys. 57, 965 (1985).

[15] G. N. Ogurtsov, Rev. Mod. Phys. 44, 1 (1972).

[16] R.K. Janev, L.P. Presnyakov, V.P. Shevelko, Physics of Highly Charged Ions (Springer, Berlin 1999).

[17] D. Mueller, L. Grisham, I. Kaganovich, R.L. Watson, V. Horvat and K.E. Zaharakis, Physics 
of Plasmas, 8, 1753 (2001).

[18] R.E. Olson, R.L. Watson, V. Horvat, and K.E. Zaharakis, Phys. Rev.A 67, 022706 (2003).

[19] R.L. Watson, Y. Peng, V. Horvat, G.J. Kim, and R.E. Olson, Phys. Rev.A 67, 022706 (2003).

[20] M. Gryzinski, Phys. Rev.A 138, 322 (1965).

[21] http://webofscience.com.

[22] G. Gillespie, J.Phys. B: Mol.Phys. 15, L729 (1982); G. Gillespie, Phys. Lett. 93A, 327 (1983).

[23] L.D. Landau and E.M. Lifshitz, Quantum Mechanics (Addison-Wesley Publishing Co., 1958).

[24] N. Bohr, K. Dan. Vidensk. Selsk. Mat.- Fys. Medd. 18, N8 (1948).

[25] I. D. Kaganovich, E. Startsev and R. C. Davidson, "Evaluation of Ionization cross sections in Energetic Ion-Atom Collisions," Proceedings of the 2001 Particle Accelerator Conference, (2001). http://accelconf.web.cern.ch/AccelConf/p01/PAPERS/TPAH314.PDF

[26] J.J. Thompson, Phil. Mag., 23, 449 (1912).

[27] E. Gerjuoy, Phys. Rev. A 148, 54 (1966).

[28] V.H. Ponce, Atomic Data and Nuclear Data Tables, 19, 63, (1977).

[29] L. Vriens, Proc.R. Soc. London, 90, 935 (1966).

[30] M. Scott Armel, Ph.D. Thesis, University of California at Berkeley (2000); http://faculty.oxy.edu/scottfunk/Sci/index.html.

[31] H. Bethe, Ann. Phys. (Leipz.) 5, 325 (1930).

[32] H.A. Bethe and R. Jackiw, Intermidiate Quantum Mechanics (The Benjamin/Cummings Publishing Company, sec.ed., 1968).

[33] M. B. Shah, D..S. Elliott and H. B. Gilbody, J.Phys. B: Mol.Phys. 20, 2481 (1987).

[34] Atomic Data for Fusion. Volume 1: Collisions of H, H2, He and Li Atoms and Ions with Atoms and Molecules (C. F. Barnett ed.) ORNL-6086 (1990).Atomic Data for Fusion. Volume 5: Collisions of Carbon and Oxygen Ions with Electrons, H, H2 and He (R. A. Phaneuf, R. K. Janev, M. S. Pindzola) ORNL-6090 (1987); http://www-cfadc.phy.ornl.gov/redbooks/.

[35] M. B. Shah and H. B. Gilbody, J. Phys. B: Mol. Phys. 15, 413 (1982).

[36] A. Kolakowska, M. S. Pindzola, and D. R. Schultz, Phys. Rev. A 59, 3588 (1999).

[37] Y.K. Kim and M. Inokuti, Phys. Rev. A 3, 665 (1971); M. Inokuti, Rev. Mod. Phys. 43, 297 (1971).

[38] V.P. Shevelko, I. Yu. Tolstikhina and Th. Stoehlker, Nucl. Instr. Meth. B 184, 295 (2001).

[39] G.H. Gillespie, Phys. Rev. A 18, 1967 (1978). 
[40] D. R. Bates and G. Griffing, Proc.Phys. Soc. London, 66, 961 (1953).

[41] N. Bohr and J. Linhard, K. Dan. Vidensk. Selsk. Mat.- Fys. Medd. 28, 1 (1954).

[42] R.E. Olson, Phys. Rev. A 18, 2464 (1978).

[43] R.K. Janev, Phys. Rev. A 18, 1810 (1983).

[44] N. Stolterfoht, R.D. DuBois and R.D. Rivarola, Electron Emission in Heavy Ion-Atom Collisions (Springer, 1997).

[45] J.M Rost and T. Pattard, Phys. Rev. A 55, R5 (1996).

[46] M. B. Shah and H. B. Gilbody, J. Phys. B: Mol. Phys. 18, 899 (1985).

[47] M. B. Shah, P. Mc.Callion and H. B. Gilbody, J. Phys. B: Mol. Phys. 22, 3037 (1989).

[48] P.D. Fainstein, V.H. Ponce, and R. D. Rivarola, J.Phys. B: Mol. Phys. 24, 3091 (1991).

[49] J.H. McGuire, Phys. Rev. A 26, 143 (1982).

[50] E.L. Duman, L.I. Men'shikov, and B.M. Smirnov, Sov.-Phys.-JETP 49, 260 (1979).

[51] V.I. Matveev, V.A. Pazdzerkii, and Kh. Yu. Rakhimov, Technical Physics 46, 512 (2001).

[52] R.E. Olson, Phys. Rev. A 27, 1871 (1983).

[53] S.Y. Ovchinnikov, Phys. Rev. A 42, 3865 (1990).

[54] D.I. Abramov, S.Y. Ovchinnikov, E.A. Solov'ev, Phys. Rev. A 42, 6366 (1990).

[55] S.Y. Ovchinnikov and J.H. Macek, Phys. Rev. Lett. 75, 2474 (1995). 


\section{External Distribution}

Plasma Research Laboratory, Australian National University, Australia

Professor I.R. Jones, Flinders University, Australia

Professor João Canalle, Instituto de Fisica DEQ/IF - UERJ, Brazil

Mr. Gerson O. Ludwig, Instituto Nacional de Pesquisas, Brazil

Dr. P.H. Sakanaka, Instituto Fisica, Brazil

The Librarian, Culham Laboratory, England

Mrs. S.A. Hutchinson, JET Library, England

Professor M.N. Bussac, Ecole Polytechnique, France

Librarian, Max-Planck-Institut für Plasmaphysik, Germany

Jolan Moldvai, Reports Library, MTA KFKI-ATKI, Hungary

Dr. P. Kaw, Institute for Plasma Research, India

Ms. P.J. Pathak, Librarian, Insitute for Plasma Research, India

Ms. Clelia De Palo, Associazione EURATOM-ENEA, Italy

Dr. G. Grosso, Instituto di Fisica del Plasma, Italy

Librarian, Naka Fusion Research Establishment, JAERI, Japan

Library, Plasma Physics Laboratory, Kyoto University, Japan

Research Information Center, National Institute for Fusion Science, Japan

Dr. O. Mitarai, Kyushu Tokai University, Japan

Dr. Jiangang Li, Institute of Plasma Physics, Chinese Academy of Sciences, People's Republic of China

Professor Yuping Huo, School of Physical Science and Technology, People's Republic of China

Library, Academia Sinica, Institute of Plasma Physics, People's Republic of China

Librarian, Institute of Physics, Chinese Academy of Sciences, People's Republic of China

Dr. S. Mirnov, TRINITI, Troitsk, Russian Federation, Russia

Dr. V.S. Strelkov, Kurchatov Institute, Russian Federation, Russia

Professor Peter Lukac, Katedra Fyziky Plazmy MFF UK, Mlynska dolina F-2, Komenskeho Univerzita, SK-842 15 Bratislava, Slovakia

Dr. G.S. Lee, Korea Basic Science Institute, South Korea

Institute for Plasma Research, University of Maryland, USA

Librarian, Fusion Energy Division, Oak Ridge National Laboratory, USA

Librarian, Institute of Fusion Studies, University of Texas, USA

Librarian, Magnetic Fusion Program, Lawrence Livermore National Laboratory, USA

Library, General Atomics, USA

Plasma Physics Group, Fusion Energy Research Program, University of California at San Diego, USA

Plasma Physics Library, Columbia University, USA

Alkesh Punjabi, Center for Fusion Research and Training, Hampton University, USA

Dr. W.M. Stacey, Fusion Research Center, Georgia Institute of Technology, USA

Dr. John Willis, U.S. Department of Energy, Office of Fusion Energy Sciences, USA

Mr. Paul H. Wright, Indianapolis, Indiana, USA 
The Princeton Plasma Physics Laboratory is operated by Princeton University under contract with the U.S. Department of Energy.

\author{
Information Services \\ Princeton Plasma Physics Laboratory \\ P.O. Box 451 \\ Princeton, NJ 08543
}

Phone: 609-243-2750

Fax: 609-243-2751

e-mail: pppl_info@pppl.gov

Internet Address: http://www.pppl.gov 